\title{
The developing landscape of combinatorial therapies of immune checkpoint blockade with DNA damage repair inhibitors for the treatment of breast and ovarian cancers
}

\author{
Lingling Zhu ${ }^{1 \dagger}$, Jiewei Liu ${ }^{1 \dagger}$, Jiang Chen ${ }^{2^{*}}$ and Qinghua Zhou ${ }^{{ }^{*}}$
}

\begin{abstract}
The use of immune checkpoint blockade (ICB) using antibodies against programmed death receptor (PD)-1, PD ligand (PD-L)-1, and cytotoxic T-lymphocyte antigen 4 (CTLA-4) has redefined the therapeutic landscape in solid tumors, including skin, lung, bladder, liver, renal, and breast tumors. However, overall response rates to ICB therapy remain limited in PD-L1-negative patients. Thus, rational and effective combination therapies will be needed to address ICB treatment resistance in these patients, as well as in PD-L1-positive patients who have progressed under ICB treatment. DNA damage repair inhibitors (DDRis) may activate T-cell responses and trigger inflammatory cytokines release and eventually immunogenic cancer cell death by amplifying DNA damage and generating immunogenic neoantigens, especially in DDR-defective tumors. DDRi may also lead to adaptive PD-L1 upregulation, providing a rationale for PD-L1/PD-1 blockade. Thus, based on preclinical evidence of efficacy and no significant overlapping toxicity, some ICB/DDRi combinations have rapidly progressed to clinical testing in breast and ovarian cancers. Here, we summarize the available clinical data on the combination of ICB with DDRi agents for treating breast and ovarian cancers and discuss the mechanisms of action and other lessons learned from translational studies conducted to date. We also review potential biomarkers to select patients most likely to respond to ICB/DDRi combination therapy.
\end{abstract}

Keywords: Immune checkpoint blockade, Breast cancer, Ovarian cancer, DNA damage repair inhibitor

\section{Background}

Remarkable progress has been made in the clinical application of cancer immunotherapies harnessing the immune system to identify and eradicate breast and ovarian tumors. The most notable example is the emergence of immune checkpoint blockade (ICB) to inhibit negative regulators of effector T-cell-mediated immunity. However, when administered alone, ICB approaches,

*Correspondence: chenjiang520@zju.edu.cn; prof_qh_zhou@126.com

${ }^{\dagger}$ Lingling Zhu, Jiewei Liu are first author and contributed equally

1 Lung Cancer Center, West China Hospital of Sichuan University,

Chengdu 610041, Sichuan Province, China

2 Department of General Surgery, Sir Run Run Shaw Hospital, Zhejiang

University, Hangzhou 310016, Zhejiang Province, China such as anti-cytotoxic T-lymphocyte-associated protein 4 (CTLA-4), anti-programmed cell death protein 1 (PD-1), and/or anti-programmed death-ligand 1 (PDL1) antibodies, generally elicit low objective response rates (ORRs), ranging from 0 to $33 \%$, which are durable only in a minority of cancer patients [1-3]. For example, ORRs for ICB monotherapy range from 18.5 to $39.4 \%$ in the most frequent malignancy in women, breast cancer (BC) [4], and from $8 \%$ to $9.6 \%$ in ovarian cancer (OC) [5]. To address this unmet need, and improve the efficacy of ICB, multiple combinatorial strategies are currently being developed, some of which include DNA damage response (DDR) inhibitors (DDRis). original author(s) and the source, provide a link to the Creative Commons licence, and indicate if changes were made. The images or other third party material in this article are included in the article's Creative Commons licence, unless indicated otherwise in a credit line to the material. If material is not included in the article's Creative Commons licence and your intended use is not permitted by statutory regulation or exceeds the permitted use, you will need to obtain permission directly from the copyright holder. To view a copy of this licence, visit http://creativecommons.org/licenses/by/4.0/. The Creative Commons Public Domain Dedication waiver (http://creativeco mmons.org/publicdomain/zero/1.0/) applies to the data made available in this article, unless otherwise stated in a credit line to the data. 
Defects in DDR genes hold the potential to function as biomarkers of response to ICB across multiple types of tumors [6]. The rationale for combining ICB with DDRi is also based on several studies that have demonstrated the critical role of DDR in the efficacy of cancer immunotherapy [7]. DDR proteins protect the integrity of the genome following DNA damage caused by endogenous factors (e.g., reactive oxygen species and errors during DNA replication) and exogenous insults (e.g., ultraviolet radiation, smoking, chemical substances) [8]. Thus, the use of DDRi might augment the tumor mutational burden (TMB), thereby increasing neoantigen production and anticancer T-cell activity [9], potentiating antitumor immunity. For example, targeting poly-ADP-ribose polymerase (PARP) markedly increased PD-L1 expression in cancer cells, augmenting the antitumor effect of PD-L1 blockade and cytotoxic T-cell infiltration in gynecologic cancers [10]. Therefore, patients with tumors carrying DNA mismatch repair (MMR) deficiency (MMRd), homologous recombination deficiency (HRD), breast cancer $1 / 2$ (BRCA1/2) genetic defects, or other defects in DDR genes [9], who present more TMB and neoantigens, can benefit from DDRi [11]. For example, MMRd is characterized by the loss of function of the MMR pathway and can generate many insertion and deletion (indel) mutations; this indel mutational load has generated a substantial number of immunogenic neoantigens, potentially driving immunotherapeutic responses [12]. Moreover, compared to homologous recombination repair (HRR), a conservative mechanism contributing to DNA double-strand break (DSB) repair, HRD, such as BRCAmutation in tumors, enhanced neoantigen burden [13].

Of note, deficiency of DDR genes occurs in a wide variety of malignancies, such as prostate, bladder, pancreatic, non-small cell lung cancers, and triple-negative $\mathrm{BC}$ (TNBC) [11]. HRD occurred in more than $20 \%$ of BCs, as well as OCs, pancreatic cancers, and gastric cancers [14] and in approximately $50 \%$ of epithelial OCs [15] and 69\% of TNBC [16]. The majority of OCs and BCs are originating from epithelial cells that undergo constant division and cyclical exposure to estrogen during the female hormonal cycle, making them vulnerable to DNA damage [17]. According to the concept of a synthetic lethality among a functional genetic defect in an HR-related gene, the combination of DDRi and ICB in BCs and OCs with HRD is promising. For instance, DDRis, namely PARP inhibitors (PARPi) [18] and checkpoint kinase 1 (CHK1) inhibitor [19], have predominantly been studied in patients with breast or ovarian cancers. Still, the interactions between ICB and DDR pathways vary, and the synergy between DDRi and ICB against cancers, independent of DDR deficiency status, needs to be further clarified according to the latest preclinical models and clinical data. For instance, in both HRD and HRR settings, clinical evidence for OCs showed a synergistic antitumor activity of PARPi in combination with ICBs [5].

Besides PARPi, other agents and factors targeting the proteins involved in the DDR pathway include ataxia telangiectasia-mutated (ATM), CHK1, ataxia telangiectasia and Rad3-related protein (ATR), DNA-dependent protein kinase (DNA-PK), WEE1, classical non-homologous end joining (cNHEJ), and alternative end joining (Alt EJ) [9, 20]. Of the solid tumors reviewed, OCs have demonstrated efficacy in the treatment of DDRi. Moreover, $\mathrm{OC}$ is the first cause of death from and the second most common gynecological malignancy [21]. To date, three PARPi, olaparib, rucaparib, and niraparib, are approved by FDA for treatment of OCs. Among them, rucaparib $[22,23]$ is approved in the relapsed setting for OC patients with BRCA mutations, olaparib for first-line maintenance treatment in newly diagnosed stage III-IV ovarian patients who are in complete or partial synergistic antitumor activity of PARP inhibitors (PARPi) in combination with ICBs [5]. response to first-line platinum-based chemotherapy or to first-line chemotherapy [24] plus bevacizumab combination [25], and olaparib $[26,27]$, rucaparib [28] as well as niraparib [29] for maintenance therapy in patients with platinum sensitive recurrent $\mathrm{OCs}$ (after $\geq 2$ lines chemotherapy), regardless of BRCA status (Table 1).

Besides OCs, TNBC is among the most lethal diseases affecting women with few targeted therapies [30]. BC is a heterogeneous disease including more than 20 histologic types, among which TNBC represents the main $\mathrm{BC}$ type with BRCA1/2 mutations [31]. Success regarding DDRi in OC treatment has paved the way for clinical trials in BCs. Two PARPi, olaparib [32] and talazoparib [33], are now approved in HER2-negative, BRCA1/2-mutant advanced BCs (Table 2). Although testing of DDRi combination with ICBs is being pursued in a growing number of clinical trials worldwide for a wide range of cancers, currently available data are inconsistent, even in patients with the same cancer type but different molecular characteristics $[34,35]$. Here, we primarily discuss how ICB, DDRi, and their combination, may impact the signaling mechanisms and tumor immune microenvironment (TME) in breast and ovarian cancers with HRD, while proposing strategies to optimize therapy. We also discuss both potential biomarkers for patient stratification and determining different toxicities of PARPi as well as distinct levels of PARP trapping [36], and outline opportunities and challenges regarding the promising combination strategies to overcome ICB resistance.

Reviewing these concepts and strategies is timely, given the expectation that an increasing number of $\mathrm{BC}$ and $\mathrm{OC}$ patients will be treated with DDRi, ICB, or their 


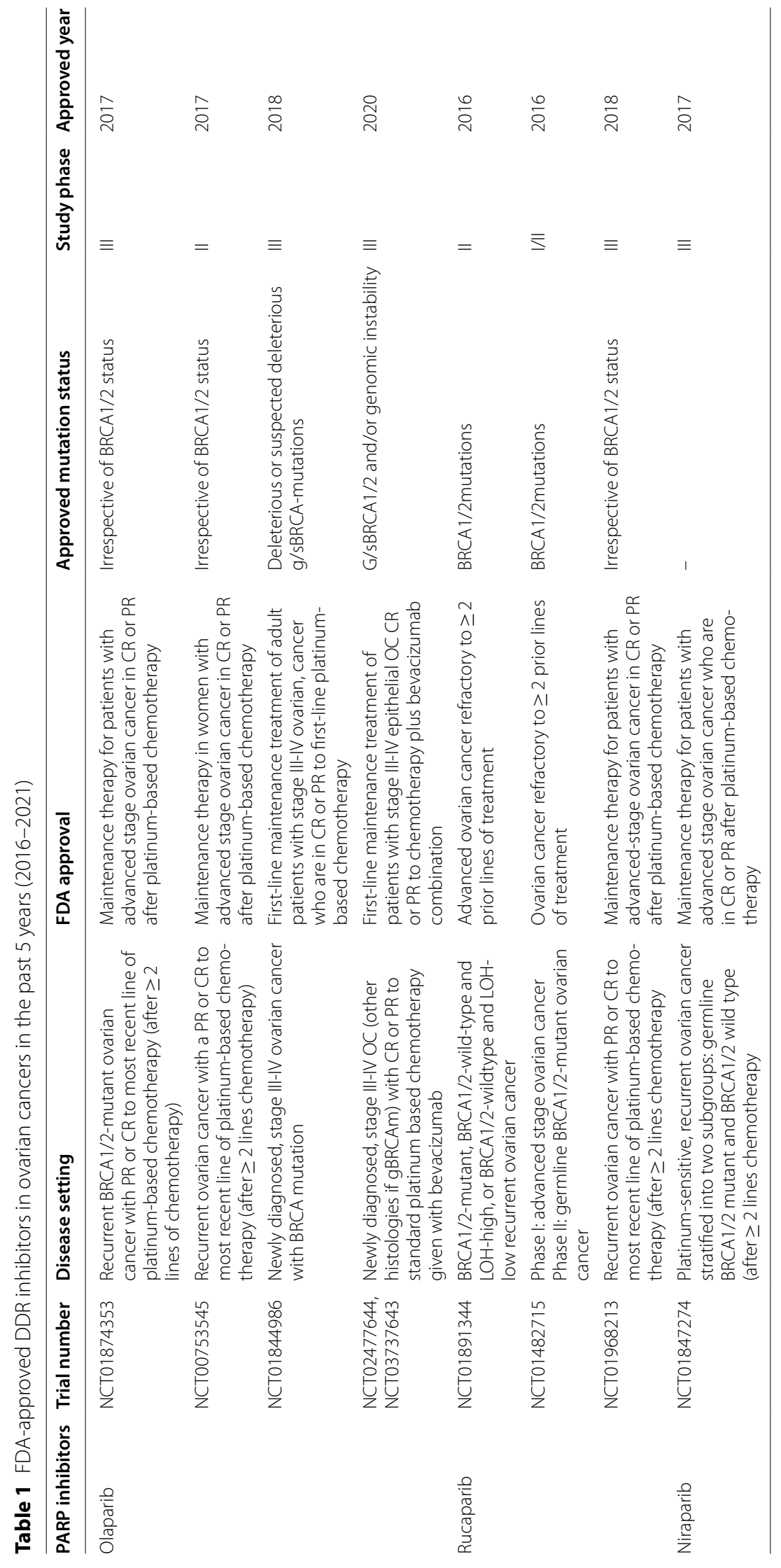


Table 2 FDA-approved DDR inhibitors in breast cancers in the past 5 years (2016-2021)

\begin{tabular}{|c|c|c|c|c|c|c|}
\hline PARP inhibitors & Trial number & Disease setting & FDA approval & $\begin{array}{l}\text { Approved mutation } \\
\text { status }\end{array}$ & Study phase & Approved year \\
\hline Olaparib & NCT02000622 & $\begin{array}{l}\text { Metastatic, gBRCA1/2- } \\
\text { mutant, HER2-negative } \\
\text { breast cancer after } \leq 2 \\
\text { prior lines of chemo- } \\
\text { therapy }\end{array}$ & $\begin{array}{l}\text { Metastatic, HER2-negative } \\
\text { breast cancers }\end{array}$ & BRCA1/2 mutations & III & 2018 \\
\hline Talazoparib & NCT01945775 & $\begin{array}{l}\text { Advanced and/or } \\
\text { metastatic HER2-negative } \\
\text { breast cancer with ger- } \\
\text { mline BRCA1/2 mutation }\end{array}$ & $\begin{array}{l}\text { HER2-negative locally } \\
\text { advanced or metastatic } \\
\text { breast cancer }\end{array}$ & $\begin{array}{l}\text { Deleterious or suspected } \\
\text { deleterious germline } \\
B R C A \text { mutation }\end{array}$ & III & 2018 \\
\hline
\end{tabular}

combination. The progress in these areas has been strikingly rapid, with FDA approvals of four PARPi and multiple immunotherapies in breast and ovarian cancers, respectively.

\section{Mechanisms of ICB resistance in solid tumors}

The major histocompatibility complex (MHC) on the cell surface displays peptides to the T cell receptors (TCR) on antigen-specific $\mathrm{T}$ cells to activate anti-tumor $\mathrm{T}$ cell responses [37]. Actions of immune checkpoints are mediated through the interactions between the ligands (B7 family members: B7-1, B7-2, PD-L1, B7-H2, B7-H3, and $\mathrm{B} 7-\mathrm{H} 4)$ and the receptors (CD28, PD-1, and CTLA4) [38]. CD28 and CTLA4, the costimulatory receptors, compete for the same ligands, B7-1 (CD80) and B7-2 (CD86) [39]. However, CTLA4 binds these ligands with higher avidity than CD28, allowing CTLA4 to compete with $\mathrm{CD} 28$ for ligand and deliver a negative regulatory signal to the T cell [39]. Currently, targeting PD-1, PD-L1, and CTLA-4 with monoclonal antibodies is the mainstay of ICB, with indications for their use in monotherapy or combination in multiple cancers [40]. Importantly, these approaches are markedly different in terms of mechanism of action. For instance, CTLA-4 inhibits T-cell activation, whereas PD-1, another key inhibitor, terminates the effector T-cell responses by interacting with its ligands PD-L1 (B7-H1) and PD-L2 (B7-DC) [41]. Compared with PD-L1, PD-L2 has a two- to six-fold higher affinity for PD-1 [42], while the PD-L1 antibodies blocking the interaction between PD-L1 and PD-1 do not affect PD-L2/ PD-1 interaction [43]. PD-L2 also binds repulsive guidance molecule $\mathrm{b}$ on macrophages, dendritic cells (DCs), and some epithelial cell types with unknown mechanism [41]. Additionally, B7-H3, one of B7 family members, can co-stimulate proliferation of both CD4+ and CD8+ $\mathrm{T}$ cells, but the specific receptor remains unknown [38] (Fig. 1). Despite considerable early successes and tolerable side effects compared to conventional treatments, such as surgery, chemotherapy, radiotherapy (RT), and targeted therapy, the overall response rates of ICB are generally limited [44], being approximately $5-23 \%$ in BCs [45] and 6-15\% in OCs [46].

The widely held view is that activation of effector T-cells $\left(T_{\text {eff }}\right)$ is the key beneficial mechanism in human tumors [47]. Thus, the prevailing hypothesis is that the efficacy of ICB is limited to patients with pre-existing antitumor immunity and $T_{\text {eff }}$ infiltration, while resistance to ICB, which is observed in most cancer patients, is mediated by barriers that impact $T_{\text {eff }}$ infiltration and/ or activity. These barriers are multifactorial and the resistance mechanisms that can be reversed by inhibition of DDR signaling pathways [48-53]. These factors may include low neoantigen expression and downregulation of MHC expression on the cancer cells, imbalance in immune checkpoint expression, increased tumor infiltration by suppressive immune cell populations, tissue hypoxia, tumor metabolic status, and immunosuppressive cytokines. Here, we focus on the resistance mechanisms related to DDR signaling pathways (Fig. 2).

\section{Low neoantigen burden}

Genomic analyses have revealed that mutational processes altering DDR and repair pathways impact responses to ICB, thus influencing clinical outcomes in patients treated with ICB $[6,54]$. Non-synonymous somatic alterations presented by tumor cells can be recognized by the immune system as non-self and neoantigens, that is, tumor-associated antigens eliciting T-cell responses [55]. Moreover, TMB serves as a surrogate marker for tumor neoantigen load, representing the number of cancer cell mutations. Meanwhile, elevated TMB was proposed to increase the chances of generating immunogenic neoantigens and the true neoantigen burden, that is, the number of alterations actually targeted by T-cells may have a stronger impact on ICB response than TMB [55]. For example, tumors with high microsatellite instability (MSI-H) accumulate considerable somatic mutations secondary to deficits in DNA MMR. The 


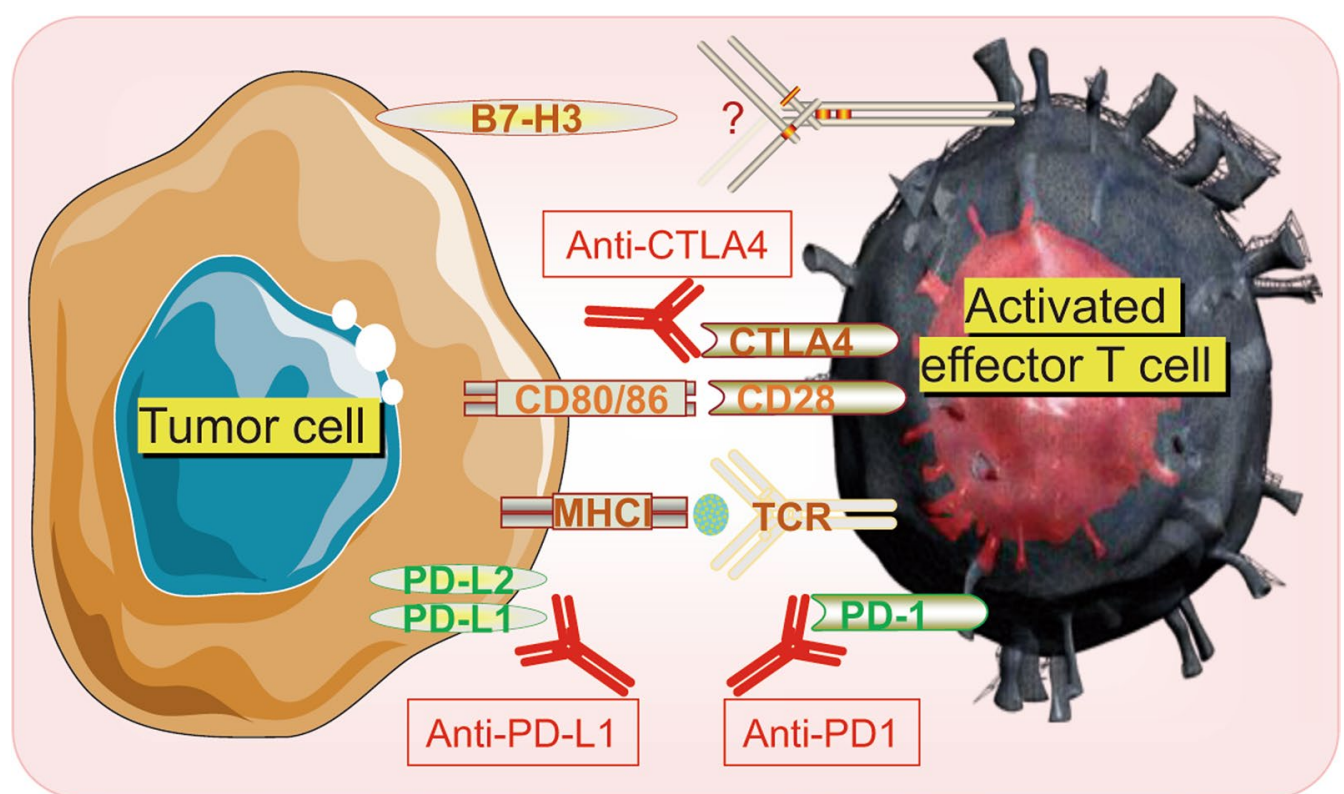

Fig. 1 Schematic illustration of tumor cells interacting with activated T cell. Inhibitory immune checkpoints, such as CTLA-4, PD-1, PD-L1 and B7-H3, bind with their partners to blockade T cell activity, while ICBs, such as anti-CTLA-4, anti-PD-1, anti-PD-L1 activate T cell by preventing the interaction cytotoxic T-lymphocyte-associated antigen 4 (CTLA-4); major histocompatibility complex I (MHCI); T cell receptor (TCR); programmed death-ligand 1 (PD-L1); programmed death-1(PD-1); immune checkpoint blockade (ICB)

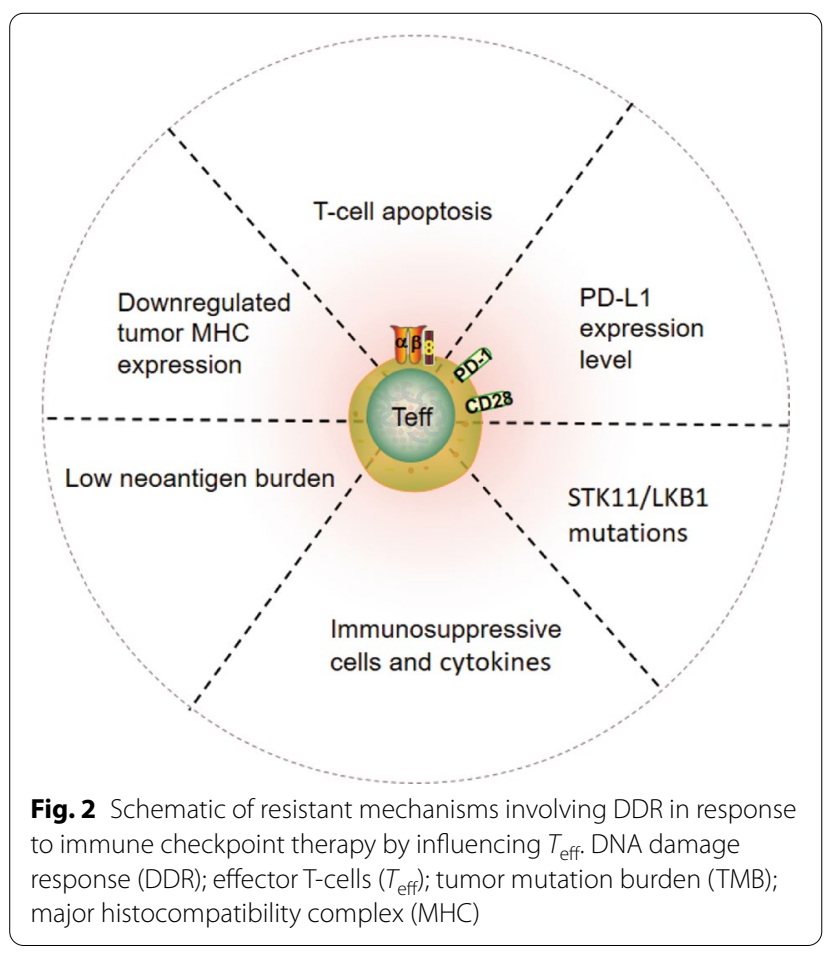

resultant high mutational burden renders tumors immunogenic and sensitive to PD-1 ICB, while many patients with MMR-proficient (pMMR) tumors fail to respond to
PD-1 blockade therapy [56]. For instance, tumors with MMRd show 20/20 (100\%) pathological response, while pMMR early stage colon cancers correlate with poor response rates with $4 / 15$ (27\%) pathological responses [57]. Despite the weaker impact of homologous recombination (HR) deficiency on TMB relative to MMRd, catastrophic DNA damage driven by DDRi can be favorable to enhance ICB therapy efficiency [49].

However, MMRd-induced mutations are more likely to be predominantly subclonal, triggering highly heterogeneous tumors [58]. Mcgranahan et al. demonstrated that subclonal (branch) neoepitopes is less effective than clonal neoantigens (trunk) in driving tumor clearance with ICB [59]. Moreover, among lung cancer patients receiving ICB treatment, tumors without certain DNA repair mutations exhibit a lower response rate relative to those with such mutational signatures $[60,61]$. Collectively, tumors with deficiencies in DDR pathways respond better to ICB due to elevated neoantigen load [48].

\section{PD-L1 expression level}

In addition to TMB, PD-L1 expression is evaluated histologically as another primary biomarker for ICB therapies in cancer patients [62]. PD-L1 expression from both malignant and immune cells can be stimulated by inflammatory cytokines, such as interferons (IFNs) and work concretely to functionally modulate the cytotoxic T-cell lymphocytes (CTLs) in the TME [63]. Moreover, 
interferon regulatory factor 7 (IRF7) enhances constitutive PD-L1 expression in an IFN- $\gamma$ independent fashion through directly inducing transcription of PD-L1 [64]. Therefore, comprehensive and dynamic evaluation of both the global PD-L1 level and IFN expression on T-cells and tumor cells, rather than monitoring only surface PD-L1 on tumor cells, should be a more effective approach for predicting responses to ICB. Additionally, DNA damage, amplified by DDRi, can induce PD-L1 expression by activating both IRF1 and IFNs, providing further rational for combinatorial ICB and DDRi therapeutic strategies [50].

\section{T-cell apoptosis}

A vital component of T-cell-mediated antitumor immune responses is the strong tumor-reactive T-cell infiltration into tumor tissues. The International tumor-infiltrating lymphocyte (TIL) Working Group has developed a standardized methodology for BC TILs used in clinical practice [65]. However, even for tumors with a substantial number of TILs prior to treatment, ICB can be rendered ineffective due to the dysfunctional T-cell phenotype [66].

T-cell apoptosis, a mechanism of tumor-induced T-cell dysfunction, can be triggered by binding of Fas, DR3, DR4, DR5, and TNFR1 on T-cells, with their respective ligands, thereby mediating immune evasion [67]. Impairing DNA repair machineries via KML001, a telomere-targeting drug, also reportedly blocks cell proliferation, cytokine production, and promotes apoptosis of T-cells via suppression of telomeric repeat binding factor 2 (TRF2), telomerase, topoisomerase I and II alpha (Top1/2a), and ATM kinase activities [68]. Moreover, KML001 triggers caspase-3-dependent T-cell apoptosis via telomeric DDR, while caspase-3 cleaves PARP during apoptosis [69]. Additionally, accelerating DNA damage by DDRi may serve to reprogram the TME inflammatory milieu [70], driving the recruitment and infiltration of T-cell into the tumor bed [49], thereby remodeling "cold tumors" to "hot tumors", via activation of the immune response, as well as dysfunctional T-cell phenotypes and subsequent apoptosis.

\section{Downregulated tumor MHC expression}

The loss or downregulation of tumor MHC-I expression constitutes a main tumor escape mechanism from T-cell-mediated immune responses via influencing the degree and composition of the immune cellular infiltration [71]. Conway et al. showed that the loss of MHC-I/ II expression contributed to resistance to ICB [62]. However, DNA damage induced by DDRi can enhance radiation-induced tumor cell MHC-I surface expression [51], supporting the use of DDRi for the reversal of ICI resistance by increasing MHC-I expression.

\section{Role of immunosuppressive cells and cytokines in tumors}

Various immune cell subpopulations are identified as protumorigenic due to their contributions toward an immunosuppressive environment, including Tregs, M2-type tumor-associated macrophages (TAMs), plasmacytoid DCs, N2-type neutrophils, and myeloid-derived suppressor cells (MDSCs) that directly or indirectly inhibit CTL responses.

CD4+CD25+Foxp3+ Tregs exert their non-specific immune suppression via modulating either T-cells or antigen presenting cells (APCs) in a cell-to-cell contactdependent manner by producing inhibitory cytokines, including IL-10 and transforming growth factor beta (TGF- $\beta$ ) [72]. In fact, AZD6738, a ATR inhibitor (ATRi), reportedly correlates with Treg infiltration in lung cancer and colorectal cancer murine models [51,73].

Macrophages are designated TAMs once they have migrated to tumors. M1 macrophages are the effector cells that participate in tumor-cell-killing role via secretion of cytokines (IL-12 among others) [74]. Alternatively, M2 TAMs promote tumor progression within the TME via hampering CD8+ T-cell responses [75]. Of note, SRA737, a CHK1 inhibitor, affects immunosuppressive M2 TAMs as well as MDSC populations via induction of immunomodulatory factors, including type I IFN $\beta$, CCL5, and CXCL10, and exerts a synergistic effect with anti-PD-1/PD-L1 therapy and low doses of gemcitabine [52]. MDSCs are a heterogeneous population of immature myeloid cells that expand in response to soluble factors generated by tumor and stromal cells and disrupt major mechanisms of antitumor immune response [76]. In fact, depletion of MDSCs in a murine BC model, following treatment with ibrutinib, an irreversible inhibitor of Bruton's tyrosine kinase, significantly improved the efficacy of immune-based therapies, including that of anti-PD-L1 therapy [76].

Taken together, immunosuppressive cells may inhibit the functionality of activated lymphocytes and induce CTL exhaustion via immunosuppressive cytokines, while DDRis have the potential to impact immunosuppressive cells.

\section{Others}

Various signaling pathways, including MAPK, JAKSTAT, PI3K-AKT, WNT- $\beta$-catenin, and Hippo pathways, highly correlate with tumor formation and evolution, while alterations in genes associated with these pathways impact the response to ICB $[62,77]$. For example, phosphatase and tensin homolog (PTEN), a suppressive gene, negatively regulates PI3K/AKT signaling and suppresses 
tumor development via dephosphorylating PIP3 [78]. Moreover, loss, inactivation, or attenuation of PTEN is the most common genomic aberration of the PI3K and interactive pathways in various types of malignancies, with PTEN loss of heterozygosity ( $\mathrm{LOH}$ ) reported in hepatocellular (57\% of patients), colorectal $(48 \%)$, gastric (36\%), prostate (52\%), and endometrial (49\%) cancers [79]. PTEN loss has also been linked to DSB repair by regulating the DDR protein RAD51, and CHK1, and has been identified as a predictive marker for PARP inhibitors [80]. Meanwhile, the ATM inhibitor KU-60019 was specifically toxic in PTEN-deficient cancer cells and tumor xenografts compared to wild-type cells [81].

A clinical trial reported that STK11/LKB1 mutations, the most prevalent genomic driver of PD-1 blockade resistance, are negatively related to therapy in KRASmutant lung adenocarcinoma [53]. Meanwhile, another study demonstrated that even in the setting of LKB1 loss, KRAS-mutant lung cancers remained treatmentrefractory and resistant to ICB [82]. Mechanistically, LKB1 loss results in silencing of stimulator of interferon genes (STING) and insensitivity to cytoplasmic doublestrand DNA sensing, while promoting immune escape. Additionally, upon cytoplasmic DSB, STING can activate $\mathrm{TBK} 1 / \mathrm{IKK} \varepsilon$, which subsequently stimulates release of cytokines/chemokines, including IL-6 and CCL5, and ultimately generates an immunosuppressive TME that impairs ICB response [82].

Taken together, increasing evidence reveals that aberrant pathway proteins related to DDR can contribute to ICB resistance in a myriad of cancers.

\section{The potential effects of DDRi on the immune system}

Impressive durable responses to ICI occur in a relatively small fraction of cancer patients, providing an opportunity to test combination strategies that will have more wide-reaching impact. Intrinsic and acquired ICB resistance has directed research toward novel combination treatment strategies aimed at transforming a higher proportion of non-responders into responders in BCs and OCs [83]. Therefore, combination of ICB with other immune-activating strategies, such as DDRi, might be a promising approach to overcome ICB resistance in cancer patients who are PD-L1-negative or PD-L1 positive but acquire resistance to ICB. Considering that DDR proteins maintain genome integrity, amplifying DNA damage using DDR pathway inhibitors may effectively increase the TMB and neoantigen production, alter the inflammatory milieu of the TME, and trigger immunogenic cancer cell death, subsequently activating antitumor immune responses (Fig. 3). Thus, combination of DDRi with ICB, such as anti-PD-1/PD-L1 or anti-CTLA-4 ICB, may initiate antitumor immunity, mediating durable tumor regression (Table 3). Here, we mainly discuss how the efficacy of ICB in the treatment of TNBC and OCs is augmented by DDR inhibition.

\section{Enhancing tumor antigen release}

Inhibition of DDR in sensitive tumor cells, such as those with MMRd, DNA modification, and replication errors, or other alterations of DDR genes including $B R C A 2$, PRKDC, RAD51C, LIG3, and RAD17, leads to accumulation of DNA damage, genomic instability, TMB, mutation-associated neoantigens, and ultimately cell death [70, 84, 85]. Mutation-associated neoantigens can activate an adaptive immune response to selectively target cancer cells. An enhanced mutation and neoantigen load may be associated with an improved T-cell response against neoantigens presented by cancer cells, which can be strengthened by ICB [85]. For example, neoantigens presented by MSI-positive tumors augment the release of IFN- $\gamma$ from TILs, particularly T-cells, thereby elevating PD-L1 levels in tumors and immune cells [86]. IFN- $\gamma$ stimulates the STAT1/2/3/IRF1 pathway by binding to the IFN- $\gamma$ receptor, thus triggering PD-L1 expression [50]. Tumors and immune cells with HRD also present enhanced neoantigen burden, TILs, and PD-1/PD-L1 level in BRCA1/2-mutated OCs [87] (Fig. 3).

BCs with germline BRCA1/2 (gBRCA1/2) mutations account for only $5-7 \%$ BC cases [88]. By contrast, highgrade TNBC is characterized by a high mutational rate [89]. Along with BRCA mutations, genomic loss of heterozygosity (LOH), large-scale translocations (LSTs), and telomeric allelic imbalance (TAI) [29], might also be molecular hallmarks of HRD [22]. Although both somatic and gBRCA $1 / 2$ aberrations, resulting in high tumor $\mathrm{TMB}$, have been approved by the FDA to function as a companion diagnostic tool for only BRCA1/2-mutated OCs [9], the antitumor activity of PARPi in BRCA wildtype tumors has been gaining attention, called "beyond BRCA" efficacy [90].

Substantial mutant neoantigens in MMRd cancers make them sensitive to ICB therapy in 12 different tumor types [91, 92], indicating a pan-tumor biomarker function for ICB efficiency. However, MMRd was only observed in around $1 \%$ of $\mathrm{BCs}$, with $1.8 \%$ in TNBC [93], indicating that its use should be restricted to high-risk individuals. Low germline MMR gene mutations (2\%) with low TMB and inconclusive evidence regarding MMRd were also noted in OCs, while a clinical relevance for immunogenic biological features and MMRd was observed among MSI subset of clear cell OCs [94], indicating that OCs do not have to be regarded as "non-immunogenic" malignancies.

In conclusion, high levels of mutations and neoantigens due to DDR inhibition [95] activate the mutation/ 


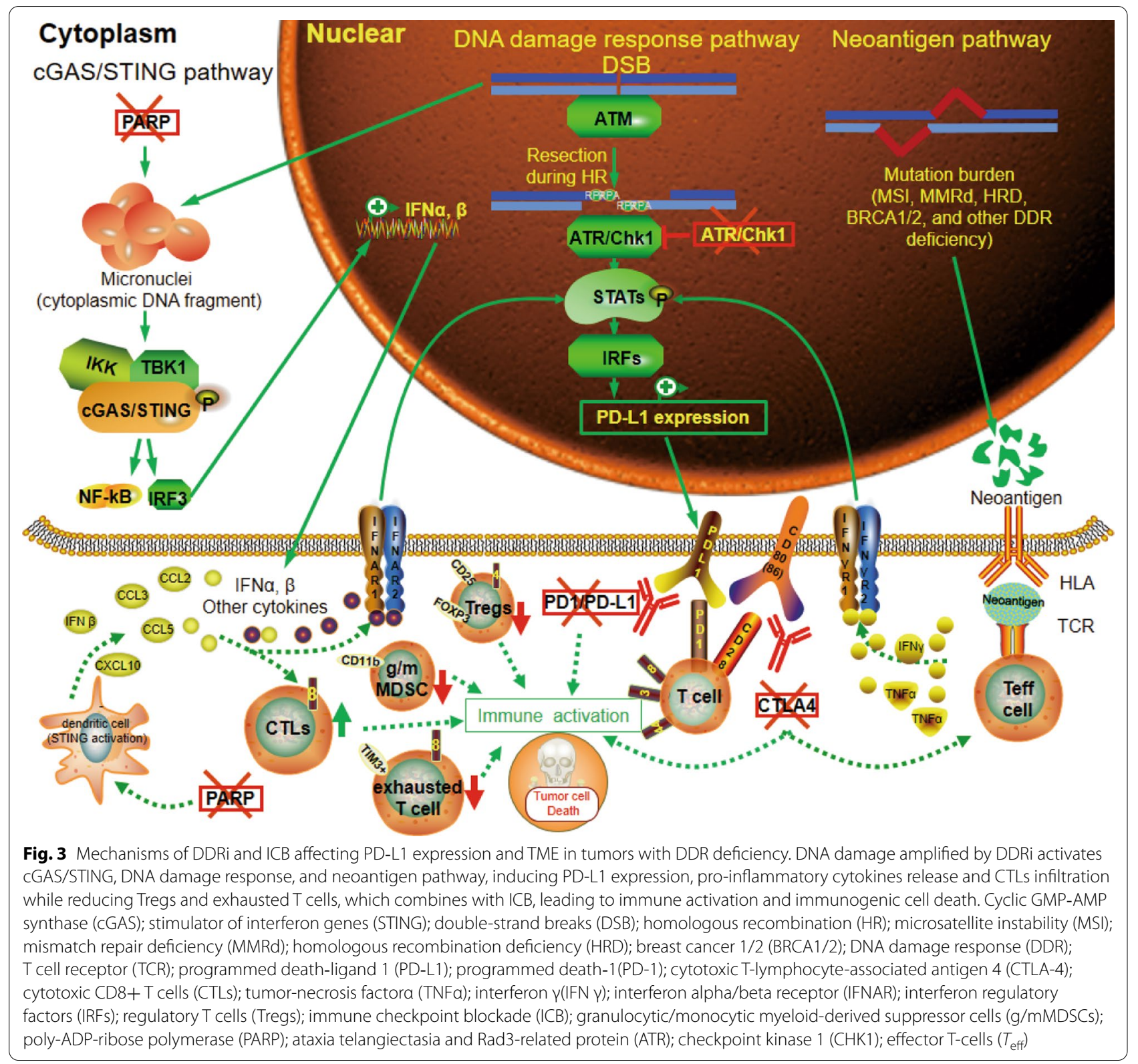

neoantigen/IFN- $\gamma$ pathway, leading to increased PD-L1 expression. To this end, genomic instability in HRD tumors potentially impacts responsiveness to DDR inhibitors when used in conjunction with ICB [96].

\section{PD-L1 expression}

In the TME, the signals from dying or already dead cells transmitted by damage-associated molecular patterns (DAMP) exposed to replicative stress or DNA damage upon DDRi not only promote immune priming, but also induce adaptive upregulation of PD-L1 levels in vitro and in vivo [97]. Mechanistically, PARPis contribute to S-phase-specific DNA damage induced by collapsed replication forks or under-replicated DNA, which subsequently induces accumulation of mitotic chromosomal bridges and micronuclei formation in the G1 phase when cells with DSBs enter mitosis $[98,99]$. Cytosolic DAMP is recognized by cyclic GMP-AMP synthase (cGAS) via generation of the second messenger, cyclic GMPAMP (cGAMP), which leads to subsequent binding and recruitment of STING in cancers treated with PARPi, such as BCs [100, 101] and BRCA1-deficient OCs [102]. STING can then recruit and activate the cytosolic kinase IKK and tank-binding kinase 1 (TBK1), contributing to the phosphorylation of STING and activation of various transcription factors, including NF-kB and IRF3, to 


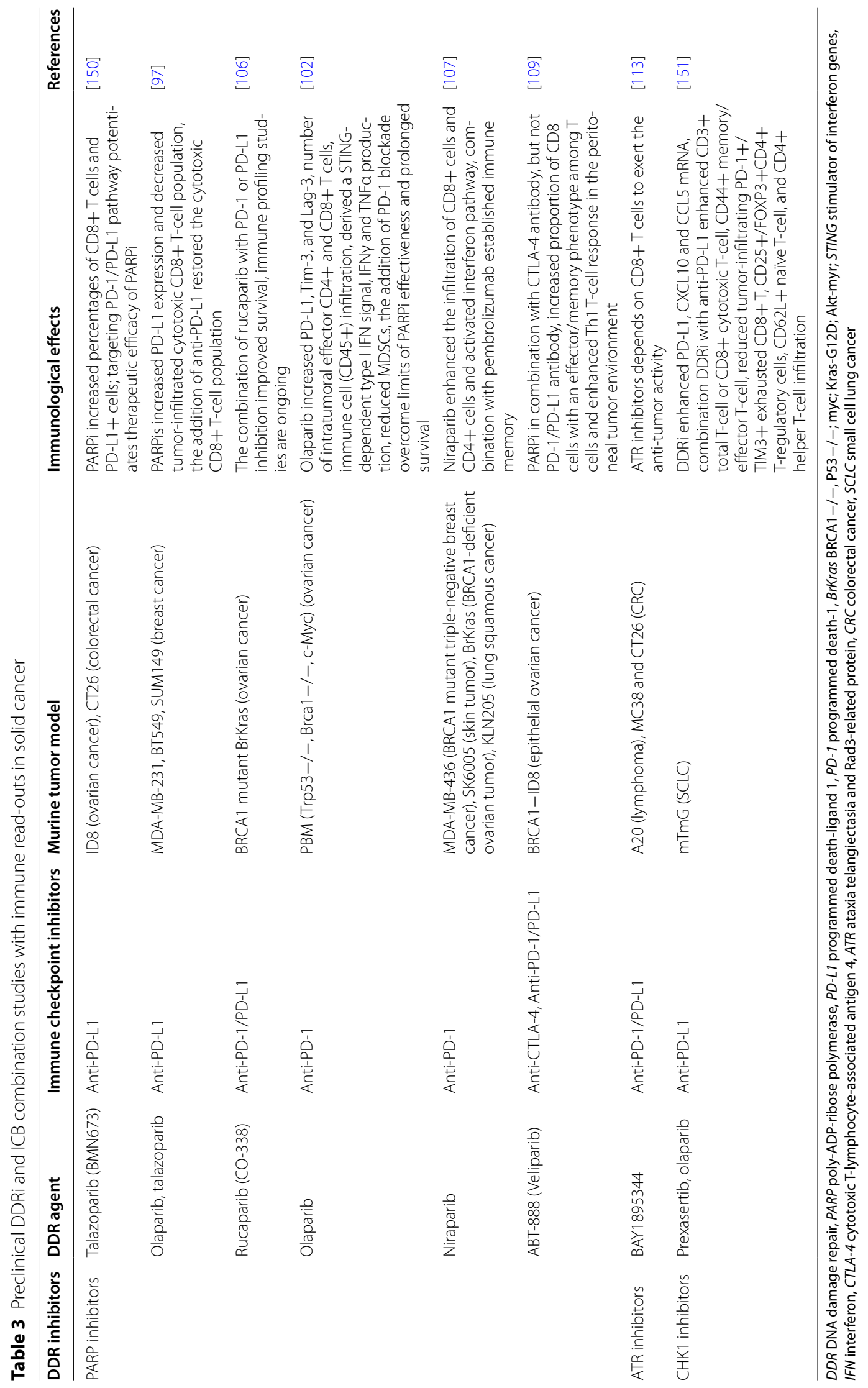


induce the expression of type I IFNs (IFN $\alpha$ and $\beta$ ), as well as other cytokines [103, 104]. Additionally, the nuclear ATM/ATR/CHK1 kinase signaling pathway can modulate PD-L1 expression following DNA damage by phosphorylating STAT1/3 (in the cytoplasm) in cancer cells [50]. When cells enter the $S / G 2$ phase during the progression of HR, ATM, a sensor of DSBs, is immediately activated at the DSB site and the DSBs undergo resection [105]. The generated ssDNA subsequently becomes coated with RPA and activates ATR/CHK1 at the ssDNA gaps [99]. Activated Chk1 is transported from the nucleus, after which it directly phosphorylates STAT $1 / 3$ and activates IRF1, which is responsible for the DSB-dependent PD-L1 upregulation [99] (Fig. 3).

This adaptive upregulation of PD-L1 can exert an immunosuppressive effect, likely blocking the PARPimediated immune activation [97]. This effect can be overcome by combining PD-1/L1 blockade with PARPi in both BCs [97] and OCs [106]. In fact, these in vitro and in vivo studies revealed that adding DDRi to ICB restores the cytotoxic CD8+ T-cells [97] and establishes immune memory [107], thereby potentiating therapeutic efficacy.

Alternatively, ATR kinase inhibitors (AZD6738 and VE-821) sensitize cancer cells to T-cell killing by downregulating the cell surface expression of PD-L1 in a proteasome-dependent manner to attenuate the PD-L1/ PD-1 interaction in MDA-MB-231 BC cells [108]. Meanwhile, in an HPV-driven malignancy model, ATRi and ATRi-RT therapy could drive PD-L1 mRNA expression primarily in $\mathrm{CD} 45+\mathrm{CD} 3-$ cells, with a relatively minor contribution in tumor cells [51]. The cause for PD-L1 upregulation by PARPi and its downregulation by ATRi may be the different mechanisms employed to activate PD-L1, as described above. Moreover, the variable PD-L1 expression upon ATRi may be due to the varying responses to DNA damage and immunogenicity in different tumor models, as well as the distinct time points chosen for analysis. However, the common feature between these two treatment strategies is their mutual ability to potentiate DNA damage, thereby enhancing antitumor immune responses.

A preclinical study in BR5 mouse OCs harboring deficient BRCA1 revealed that the CTLA-4 antibody, and not PD-1/PD-L1 blockade, together with the PARPi, veliparib, resulted in immune-mediated tumor remission and long-term survival $(p<0.0001)$ by increasing CTLs with an effector/memory phenotype and inducing IFN- $\gamma$ and TNF $\alpha$ expression by promoting the Th1 effector phenotype among T-cells [109]. One reason for these contradictory findings is the use of distinct models, with differences in their TME. Another explanation for the selective efficacy of CTLA-4 blockade is that the activation of new lymphocyte clones, instead of the reversal of T-cell exhaustion, contributes to immune-mediated antitumor responses in the BRCA1 model compared to PD-1 blockade [110]. This difference may also result from using different PARPi in terms of the catalytic inhibition and PARP trapping potencies, with veliparib displaying a weaker efficacy in HRD and HR-proficient (HRR) cell lines compared with olaparib, talazoparib, or rucaparib $[111,112]$.

\section{Reprogramming the TME}

CTLs are the key mediators of antitumor immunity [85]. DDRi could have immunosuppressive effects or improve the antitumor response by influencing CTLs [97]. T-cell priming might rely on IFN- $\beta$ activation via the STING protein complex to generate an antitumor immune response [85]. For example, olaparib elicits an antitumor immune response by inducing both intra-tumoral and peripheral effector CD4+ and CD8+ T-cells. Upon PARP inhibition, APCs, such as DCs, stimulate a STINGdependent type I IFN signal (IFN- $\beta$ ) and CXCL10 secretion, which are partially responsible for the treatment efficacy of PARPi in a co-culture system of BRCA1deficient OCs [102]. Besides, olaparib promoted CD8+ T-cell recruitment via activating cGAS/STING signaling in tumor cells with paracrine activation of DCs; this was more obvious in HR-deficient than in HRR TNBC cells and in vivo mouse models [100]. An ATR inhibitor, BAY1895344, enhances antitumor efficacy following PD-1/L1 treatment relying on CD8+ T-cells [113]. Notably, the augmented antitumor efficacy is sequencedependent and has only been achieved post-application of PD-1/PD-L1 blockade, followed by BAY 1895344 treatment (Fig. 3).

PARPis also reportedly decrease the tumor-infiltrating cytotoxic CD8+ T-cell population, while addition of PD-L1 blockade increases the cytotoxic CD8+ T-cell population, thereby re-sensitizing PARPi-treated tumor cells to T-cell killing in TNBC [97]. Mechanistically, PARPis enhance the level of PD-L1 expression on EMT6 tumor cells, a TNBC cell line, by inactivating the GSK3 $\beta$ pathway in vitro and in vivo, when inoculated into a syngeneic mouse model. This upregulation induces a decline in the tumor-infiltrating cytotoxic CD8+ T-cell population, as determined by quantifying the level of IFN- $\gamma$. Hence, neoantigen-specific tumor-infiltrating T-cells become subjected to ICB modulations and are highly heterogeneous [114].

DDRi are also capable of transforming chronic, weak DNA damage, to a more robust level of damage by resetting the inflammatory microenvironment of tumors (Fig. 3). ATR inhibition enhances radiation-induced inflammatory IFN response and cytokine gene expression, either in vivo (particularly CCL2, CCL5, and 
CXCL10) or in vitro (CCL3, CCL5, and CXCL10) [51]. Inflammatory cytokines increase over days, are driven by DSB formation, and modify the TME by recruiting immune cells and are proven to be key to both local and systemic (abscopal) tumor responses [115]. Meanwhile, micronuclei with cGAS accumulation may initiate inflammatory signaling after RT [115].

Cytokine secretion into the extracellular space exerts a bystander effect on neighboring cells, contributing to an immunogenic TME. For instance, BC cells with DDR deficiency have been linked to increased production of CXCL10 and CCL5, compared to DDR-proficient cells, both of which are important for the chemotaxis of peripheral blood mononuclear cells [101]. HRD and/or DDRi might promote immunological vulnerabilities in tumors, while simultaneously inducing immunosuppressive pathways, providing a rationale for combination with ICB therapy.

Taken together, DNA damage enhanced via DDRi yields greater mutational burden, increases neoantigen expression, triggers the release of pro- inflammatory mediators, and leads to greater immune recognition of the tumor, thus increasing the level of inflammatory cytokines and TILs.

\section{Biological role of DNA damage amplified by DDRi in cancer cells}

Mutation of genes involved in the DNA MMR pathway, as well as in other DNA damage repair pathways, is reportedly enriched in patients who exhibit durable clinical benefit from ICBs [9]. Thus, using targeted agents for DDR pathways, including inhibitors of ATR/ CHK1 [116], PARP [117], ATM, cyclin-dependent kinase 4/6 (CDK4/6), DNA-PK, WEE1, and aurora kinase B (AURKB) [9] opens new exciting avenues for clinical development of ICB for cancer treatment.

DDR is a complex network of signaling pathways involving DNA damage repair, cell cycle checkpoints, and apoptosis [118] that is key to ensuring overall genomic stability and cell viability, and is responsible for repairing the two primary forms of DNA damage: single-strand breaks (SSB) and DSB.

In response to DSB, the Mre11-Rad50-Nbs1 complex stimulates the ATM-CHK2 pathway, stabilizing p53 via phosphorylation, and causing G1 arrest in normal cells [116]. However, due to frequent inactivation of p53 or retinoblastoma (RB) proteins, most cancer cells exhibit dysregulated G1 checkpoints with insufficient time for DDR to occur prior to DNA replication, causing cells to rely exclusively on intra $\mathrm{S}$ and $\mathrm{G} 2 / \mathrm{M}$ checkpoints activated by the ATR-CHK1 pathway upon stalled replication forks [99]. Hence, therapeutic inhibition targeting ATR has been shown to increase selective killing of tumor cells [116]. Cancer cells may reportedly depend on the ATR-CHK1 signaling pathway and intact S/G2-M checkpoints for repairing DNA damage [116], and thus, ATR inhibition may result in selective cytotoxic DNA damage and detrimental mitotic catastrophe to tumor cells, while normal cells with a functional G1 checkpoint will be unaffected. This also partially explains the essential nature of ATR and/or CHK1 protein kinase for regulating replication stress and ensuring genome integrity and cell survival $[119,120]$.

Moreover, WEE1 activates the G2/M cell cycle checkpoint by suppressing CDK1/2, while WEE1 inhibition abrogates the G2 checkpoint, resulting in unscheduled entry into mitosis, elevated replication stress, and subsequent nucleotide starvation and genomic defects [9].

Following SSB, PARP enzymes, key eukaryotic stress sensors, can activate various downstream proteins that participate in SSB repair or base excision repair [9]. Hence, PARP inhibition generates persistent SSB, which ultimately leads to DSB, thereby accelerating replication progression and limiting the ability of the cell to stall DNA replication and repair. This effect is particularly pronounced in cells harboring BRCA1/2 mutations, in which HR is defective, while in cells without defective HR, accumulated DSBs can be repaired by HR or NHEJ [11]. Notably, the antitumor effects of different PARPi varied due to altered PARP trapping activity, that is, the ability of PARPi to trap PARP-1 on DNA to enhance the stability of PARP-DNA complexes [36].

DNA-PK, another sensor of DNA damage, is also a critical enzyme for DNA repair via NHEJ [9]. Therefore, disrupting PARP, ATM, ATR/CHK1, WEE1, or DNAPK activity may selectively contribute to accumulation of DNA damage in tumors, sensitize cancer cells to killing, and ultimately induce cell death. Importantly, DNA repair defects are more abundant and specific in malignant tissues than in normal tissues [35, 121]. Moreover, DNA damage proteins and cancer cells have specific properties, including cancer-specific DDR defects and lack of G1 checkpoint control, that may highlight particular vulnerabilities of the cancer [122], supporting PARP, ATR, ATM, CHK1/2, and WEE1 as therapeutic targets.

Inhibition of specific DDR proteins, which normally halt the cell cycle in DNA damage repair pathways, may play various roles in different physiological and/ or pathological events, particularly during neoplasia. For example, PARPi inhibit metastatic action and tumor recurrence via modulating the hypoxic response and suppressing proliferation, epithelial-mesenchymal transition (EMT), angiogenesis, and cancer stem like cells across different tumor settings [123]. Moreover, inhibition of the ATR/CHK1 signaling pathway blocks EMT, cell proliferation, invasion, migration, tumorigenicity, and lymph 
node metastasis, while inducing apoptosis in cervical cancer [124]. Certain DNA damage sensors, such as ATM, ATR, FOXO3a and p53, function as critical regulators of autophagy and are critical to the maintenance of cell cycle arrest and DNA repair activities in histiocytic lymphoma cells [125]. Conversely, in most malignancies, autophagy can support DNA synthesis, thereby conferring survival to tumor cells under replication stress [126], whereas autophagy inhibition with niraparib treatment, a PARPi, accelerates DNA damage and cell death in laryngeal squamous cell carcinoma [127]. Moreover, DNA damage-induced senescence physiologically and pathologically correlates with aging and age-related diseases in vivo [128]. For instance, PARPi can induce senescence in BC and high-grade serous epithelial OC cells [129]. Additionally, AURKB inhibitors trigger senescence in non-small cell lung cancer cells with acquired mutations via the ATM/Chk2 DDR [130]. Nevertheless, in patients with breast tumors, low expression of XRCC1, ATM, and BRCA1 correlates with high proliferation indexes, higher tumor grade, and the presence of dedifferentiated cells [131]. Taken together, DDRi suppress adhesion, proliferation, migration, invasion, EMT, formation of autophagosome, and angiogenesis [132], while stimulating apoptosis and senescence by directly targeting DNA damage repair processes and augmenting DNA damage in cancer cells. Moreover, DNA damage, while tumorsuppressive, promotes pro-carcinogenic effects mediated by the TME (Fig. 4).

\section{Clinical translation of DDRi plus ICB as a strategy to overcome immunotherapy resistance}

Based on these promising data on the combination of DDRi with ICB therapy in preclinical and translational settings, multiple clinical trials are currently ongoing (Additional file 1: Table S1), with results for certain DDRi/ICB combinations having already been reported, including olaparib/durvalumab [133-135], niraparib/ pembrolizumab [136], pembrolizumab/olaparib [137], BGB-A317/BGB-290 [138], and AZD6738/durvalumab [139].

The first clinical trial reporting durvalumab plus olaparib demonstrated that the combination therapy had no overlapping toxicity, and the efficiency was established in two patients with a partial response (PR) and eight with stable disease (SD), yielding an ORR of $14 \%$ in 35 patients and a disease control rate of $83 \%$ in 12 patients harboring advanced-stage BCs [134]. Among them, only a small population achieved a durable response, with a clinical benefit rate (PR $+\mathrm{SD} \geqslant 6$ months) of $34 \%$. The TOPACIO/KEYNOTE-162 trial showed that the combination of niraparib with pembrolizumab exhibited a good tolerability profile, with the ORR ( $47 \%$ vs. $11 \%)$ and progression-free survival (PFS) (8.3 months vs. 2.1 months) being higher in BRCA1/2 mutant TNBC patients than in non-mutant ones [140]. Likewise, the MEDIOLA trial showed that in one of the four cohorts (NCT02734004), olaparib plus durvalumab achieved an impressive DCR of $80 \%$ and an ORR (all PRs) of $52 \%$ for patients with advanced-stage $g B R C A 1 / 2$-mutant BCs, with no obvious overlapping toxicities [141].

In the MEDIOLA trial of a gBRCA1/2-mutated platinum-sensitive relapsed OC cohort, combination of durvalumab with olaparib also exhibited no overlapping toxicity, with ORR of $71.9 \%$, median PFS of 11.1 months, and 28-week DCR of 65.9\% [142]. Meanwhile, in the TOPACIO/KEYNOTE-162 trial of an advanced platinum-resistant $\mathrm{OC}$ cohort, niraparib plus pembrolizumab achieved a DCR of $68 \%$ in the general study population, similar to the $73 \%$ DCR in patients with $B R C A 1 / 2$ mutations [140], indicating that PARPi plus ICB exhibits antitumor activity, regardless of HR or BRCA status. The combination in both trials was safe with the most common grade 3 or greater AEs being anemia (21\% TOPACIO; 9\% MEDIOLA) [5]. Notably, a phase I/II trial (NCT02953457) is testing dual blockade of the immune checkpoint using anti-PA-L1 and anti-CTLA4 (durvalumab and tremelimumab) plus PARPi (olaparib) in recurrent or refractory BRCA1/BRAC2-mutated epithelial OCs.

Combination of ICB and DDR inhibitors with other agents, including RT, chemotherapy, anti-angiogenic or epigenetic drugs, is emerging in preclinical and clinical studies based on the good tolerability of the DDRi/ ICB combination. RT can kill cancer cells, while simultaneously inducing the release of pro-inflammatory mediators, increasing infiltration of immune cells, and promoting the expression of neoantigens [84]. Meanwhile, DDRi can further enhance the immunogenic effects of RT through augmenting CTL infiltration into the tumor bed, as well as the expression and secretion of chemokines [109], while also promoting PD-1/ PD-L1 expression. Therefore, adding ICB to this combination could counterbalance such immunosuppressive effects [143, 144], providing a preclinical rationale for triple combination therapy to improve treatment efficiency of PD-axis ICB in clinical trials. A phase II study (NCT04690855) combining talazoparib (PARPi), RT, and atezolizumab (anti-PD-L1) is currently recruiting gBRCA $1 / 2$ negative patients with PD-L1+ metastatic TNBC. Additionally, more detailed studies on the clinical combinations of RT, DDRi, and ICB are warranted to address concerns regarding subclonal neoantigen generation [84].

Addition of epigenetic drugs to PARPi/anti-PD1/ PD-L1 is a latently synergistic strategy for potentiating immunogenic cell death and overcoming resistance 


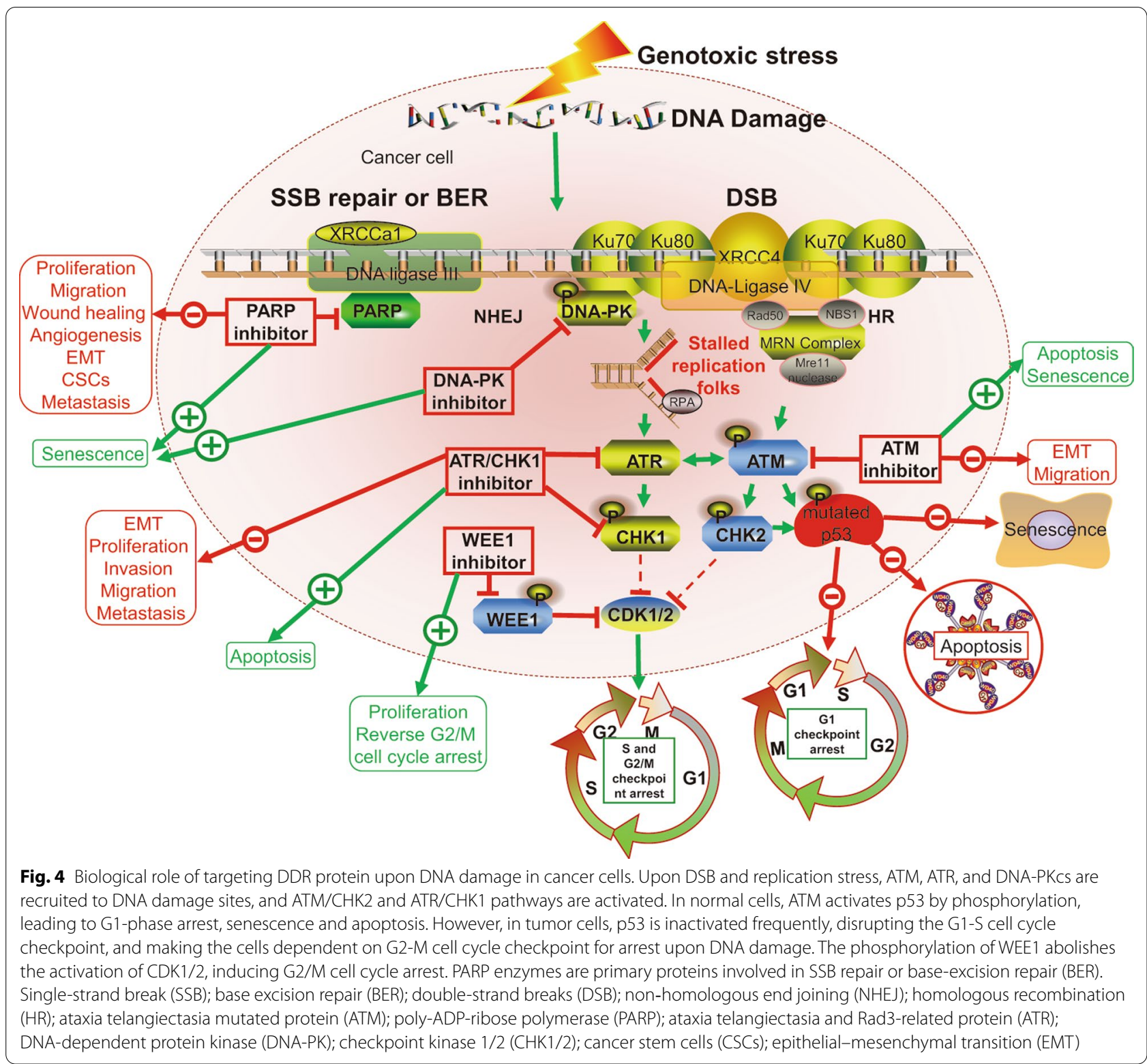

due to the additional suppression of cancer stem cells in TNBC [145]. The I-SPY2 trial reported a significantly enhanced pathologic complete response when durvalumab and olaparib were combined with paclitaxel in comparison with chemotherapy alone among women with stage II/III high-risk, HER2-negative BC; improvement was observed in both the HR+ and TNBC subsets [146]. Moreover, clinical trials of evaluating addition of bevacizumab (anti-VEGF humanized monoclonal antibody) to nivolumab plus rucaparib (NCT02873962) or to olaparib plus durvalumab (NCT02734004), niraparib plus dostarlimab (TSR-042, anti-PD1) (NCT03574779 and NCT03806049) are recruiting patients with relapsed or advanced OCs.

Other clinical trials are currently ongoing to investigate the combination of ICB with DDRis, such as durvalumab plus olaparib, AZD6738, or AZD1775, tremelimumab plus olaparib, pembrolizumab plus olaparib, or niraparib, nivolumab plus niraparib, or veliparib, atezolizumab plus olaparib, niraparib, ipilimumab plus niraparib, and BGB-A317 plus BGB-290. Taken together, the effects of these combinations are being evaluated and results are eagerly awaited. 


\section{Biomarkers for predicting clinical benefits of combination DDRi and ICB}

Biomarkers for $\mathrm{BC}$ and $\mathrm{OC}$ treatment response to DDRi plus ICB are limited. In HER2-negative stage II/III BC patients treated with triple combination (durvalumab, olaparib, and chemotherapy) compared with chemotherapy alone, a higher expression of immune gene signatures and PD-L1 mRNA expression was associated with higher pathologic complete response rates [146]. Meanwhile, mutational signature 3, reflecting HRD, and positive immune score, serving as a substitute of interferon-primed CD8-exhausted effector T-cells detected by targeted gene panel sequencing, function as a positive predictive or prognostic marker for platinum-resistant OCs when combining niraparib with pembrolizumab [147]. Further, enhanced systemic IFN $\gamma$ linked to higher PFS (HR 0.37, $p=0.023$ ) and high post-treatment levels of VEGF3R3 correlated with worse PFS in epithelial OC patients treated with olaparib plus durvalumab [10]. In other solid cancer types, there are some predictive and prognostic markers overlapping with biomarkers for ICB and DDRi monotherapy (Table 4).

As 15\% of unselected TNBC [148] and 17\% of highgrade serous OC [90] patients harbor gBRCA mutations,
BRCA1/2 alterations resulting in HRD can function as a promising biomarker for DDRi use in both OCs and BCs [149]. However, the use of these genomic aberrations as biomarkers to identify patients who are more likely to benefit from the combination therapy will require extensive validation in large and well-designed clinical trials. Ideally, future studies should integrate the complexity of the biology of both the tumor and its TME (such as PD-L1 level, CD8 T-cell infiltration, and other immune infiltrates) or gene expression profiling of genomic alterations using NGS platforms to establish a deeper understanding of the DNA damage and immune-related biomarker groups, and thus help to precisely guide the clinical development of this new strategy.

\section{Conclusions and future perspectives}

DDRi might generate immunological vulnerabilities in tumors, while concurrently stimulating immunosuppressive signaling, such as PD-1/PD-L1 signaling. Therefore, the combination of ICB with DDRi might overcome ICB resistance, inducing robust antitumor immune responses and immunogenic cell death of cancer cells, resulting in a potential cure, especially in cancers with high incidence of HRD, such as BRCA-mutant BCs and OCs. Moreover,

Table 4 Biomarkers that predict response to DDR-targeted therapies in combination with ICB

\begin{tabular}{|c|c|c|c|c|c|c|c|c|}
\hline Factor & Agents & Incidence (\%) & $\begin{array}{l}\text { Validated in } \\
\text { clinical trial? }\end{array}$ & $\begin{array}{l}\text { Association } \\
\text { with } \\
\text { favorable } \\
\text { clinical } \\
\text { outcome }\end{array}$ & $\begin{array}{l}\text { Predictive } \\
\text { versus } \\
\text { prognostic }\end{array}$ & Cancer type & $\begin{array}{l}\text { Tissue } \\
\text { type for } \\
\text { biomarker } \\
\text { assessment }\end{array}$ & $\begin{array}{l}\text { Possible } \\
\text { assay type } \\
\text { for biomarker } \\
\text { assessment }\end{array}$ \\
\hline $\begin{array}{l}\text { Mutational } \\
\text { signature } 3 \\
\text { reflecting } \\
\text { HRD, IS }\end{array}$ & $\begin{array}{l}\text { Niraparib, } \\
\text { pembroli- } \\
\text { zumab }\end{array}$ & $51 \%$ & $\begin{array}{l}\text { I/II } \\
\text { (NCT02657889) }\end{array}$ & Positive & $\begin{array}{l}\text { Prognostic, } \\
\text { predictive, or } \\
\text { both }\end{array}$ & $\begin{array}{l}\text { Ovarian } \\
\text { cancer }\end{array}$ & Tumor tissue & $\begin{array}{l}\text { Targeted gene } \\
\text { panel sequenc- } \\
\text { ing }\end{array}$ \\
\hline $\begin{array}{l}\text { Pre-existing } \\
\text { CD8+ T-cell } \\
\text { infiltrates }\end{array}$ & $\begin{array}{l}\text { Durvalumab, } \\
\text { olaparib }\end{array}$ & NR & $\begin{array}{l}\text { ॥ } \\
\text { (NCT02484404) }\end{array}$ & Positive & Predictive & Relapsed SCLC & Tumor tissue & $\begin{array}{l}\text { Immunohisto- } \\
\text { chemistry }\end{array}$ \\
\hline $\begin{array}{l}\text { MDSCs ( } \leq \text { the } \\
\text { median) }\end{array}$ & $\begin{array}{l}\text { Durvalumab, } \\
\text { olaparib }\end{array}$ & NR & $\begin{array}{l}\text { I/II } \\
\text { (NCT02484404) }\end{array}$ & Negative & Prognostic & MCRPC & Blood & $\begin{array}{l}\text { Multiparametric } \\
\text { flow cytometry }\end{array}$ \\
\hline CTC & $\begin{array}{l}\text { Durvalumab, } \\
\text { olaparib }\end{array}$ & NR & $\begin{array}{l}\text { I/II } \\
\text { (NCT02484404) }\end{array}$ & Positive & Prognostic & MCRPC & Blood & $\begin{array}{l}\text { Multiparametric } \\
\text { flow cytometry }\end{array}$ \\
\hline $\begin{array}{l}\text { CD83 } \\
\text { expression } \\
\text { on CD141+ } \\
\text { mDCs, } \\
>\text { median } \\
\text { percentage of } \\
\text { Ki67+ PD-1+ } \\
\text { cells among } \\
\text { total CD8+/ } \\
\text { CD4+ T } \\
\text { cells, }>\text { median } \\
\text { percentage of } \\
\text { Ki67+ HLA-DR } \\
\text { CD8+ and } \\
\text { CD4+ T cells }\end{array}$ & $\begin{array}{l}\text { Durvalumab, } \\
\text { olaparib }\end{array}$ & NR & $\begin{array}{l}\text { I/II } \\
(\mathrm{NCT02484404)}\end{array}$ & Positive & Prognostic & $\mathrm{mCRPC}$ & Blood & $\begin{array}{l}\text { Multiparametric } \\
\text { flow cytometry }\end{array}$ \\
\hline
\end{tabular}


DDRi plus ICBs demonstrated enhanced treatment efficiency in $\mathrm{BC}$ and $\mathrm{OC}$ patients compared with monotherapy in both first-line and recurrent settings, which primarily depended on early endpoints, such as ORR, making it suitable for patients with limited responsiveness rather than those with high response rate. Therefore, it might be more reasonable to assess clinical benefit in terms of long-term benefits, such as duration of response or OS.

Clinical trials exploring the dual combination in the neoadjuvant setting of BCs have already begun. A phase II trial (NCT04584255) is trying to investigate the synergistic efficacy of niraparib plus dostarlimab in BRCAmutated BCs and TNBC. However, more effective therapies are still required for BRCA-negative and PDL$\mathrm{L} 1$ negative $\mathrm{BCs}$, as well as non-TNBC subtypes and in the neoadjuvant setting.

In OC patients with both repair-deficient and -proficient status, ongoing phase III studies are trying to explore rucaparib plus nivolumab (ATHENA, NCT03522246), atezolizumab plus niraparib (ANITA, NCT03598270), avelumab plus talazoparib (JAVELIN, NCT03642132), and niraparib plus dostarlimab (antiPD1) (NCT03602859) in both the frontline treatment and the maintenance setting following platinum-based chemotherapy. These trials may further help answer the question of whether the combinational treatment is confined to HR-deficient or can be extended to the HRR phenotype.

Overall, the combination of DDRi with ICB is promising due to their distinct, mostly non-overlapping toxicities, particularly in BC and OC patients with HRD. However, greater clarity is needed via basic and translational studies to elucidate the mechanism of action of both DDRi and ICB as monotherapies, and in combination. Besides evaluating safety and efficacy in clinical research, validating predictive biomarkers to identify sensitive patients should become a priority.

\footnotetext{
Abbreviations

ICB: Immune checkpoint blockade; PD-1: Programmed death receptor 1; PD-L1: Programmed death-ligand 1; CTLA-4: Cytotoxic T-lymphocyte antigen 4; ORRs: Objective response rates; BC: Breast cancers; OC: Ovarian cancers; DDR: DNA damage response; DDRi: DNA damaging response inhibitors; TMB: Tumor mutational burden; PARP: Poly-ADP-ribose polymerase; MMR: DNA mismatch repair; MMRd: MMR deficiency; HRD: Homologous recombination deficiency; BRCA1/2: Breast cancer 1/2; Indel: Insertion and deletion; DSB: DNA double-strand breaks; TNBC: Triple-negative breast cancer; PARPi: PARP inhibitors; CHK1: Checkpoint kinase 1; HRR: HR-proficient; ATM: Ataxia telangiectasia-mutated; ATR: Ataxia telangiectasia and Rad3-related protein; DNA-PK: DNA-dependent protein kinase; FDA: Food and Drug Administration; TME:Tumor immune microenvironment; MHC: Major histocompatibility complex; DCs: Dendritic cells; RT: Radiotherapy; $T_{\text {eff: }}$ Effector T-cells; MSI: Microsatellite instability; pMMR: MMR-proficient; TIL:Tumor-infiltrating lymphocyte; IFNs: Interferons; CTLs: Cytotoxic T-cell lymphocytes; TIL:Tumor-infiltrating lymphocyte; TAMs: Tumor-associated macrophages; MDSCs: Myeloid-derived suppressor cells; APCs: Antigen presenting cells; ATRi: ATR inhibitor; gBRCA1/2:
}

Germline BRCA1/2; STING: Stimulator of interferon genes; CGAS: Cyclic GMP-AMP synthase; SSB: Single-strand breaks; EMT: Epithelial-mesenchymal transition; SD: Stable disease.

\section{Supplementary Information}

The online version contains supplementary material available at https://doi. org/10.1186/s13045-021-01218-8.

Additional file 1. Table S1: Summary of Clinical Studies Combining Inhibitors Targeting DDR and ICB in Solid Cancer (ClinicalTrials.gov).

\section{Acknowledgements}

We sincerely appreciate Yan Wang and Ting Cao from the Core Facility of West China Hospital, Honglin Gao and Lin Li from the Key Laboratory of Drug Targeting and Drug Delivery System of the Education Ministry of Sichuan University, Li Wang from Lung Cancer Center of West China Hospital, and Yang Yang and Xiaoting Chen from the Animal Experimental Center of West China Hospital for their assistance and suggestions.

\section{Authors' contributions}

LLZ and JWL collected the related papers and drafted the manuscript. LLZ and JWL revised the manuscript and figures. JC and $\mathrm{QHZ}$ participated in the design, revised, and finalized the manuscript. All authors read and approved the final manuscript.

\section{Funding}

Not applicable.

Availability of data and materials

Not applicable.

\section{Declarations}

Ethics approval and consent to participate Not applicable.

\section{Consent for publication}

Not applicable.

\section{Competing interests}

The authors declare no conflicts of interest in this work.

Received: 25 September 2021 Accepted: 5 December 2021

Published online: 20 December 2021

References

1. Ott PA, Bang YJ, Piha-Paul SA, Razak ARA, Bennouna J, Soria JC, et al. T-cell-inflamed gene-expression profile, programmed death ligand 1 expression, and tumor mutational burden predict efficacy in patients treated with pembrolizumab across 20 cancers: KEYNOTE-028. J Clin Oncol. 2019:37(4):318-27.

2. Christofi T, Baritaki S, Falzone L, Libra M, Zaravinos A. Current perspectives in cancer immunotherapy. Cancers (Basel). 2019;11(10):66.

3. Magaway C, Kim E, Jacinto E. Targeting mTOR and metabolism in cancer: lessons and innovations. Cells. 2019;8(12):66.

4. Lazarus G, Audrey J, Iskandar AWB. Efficacy and safety profiles of programmed cell death-1/programmed cell death ligand-1 inhibitors in the treatment of triple-negative breast cancer: a comprehensive systematic review. Oncol Rev. 2019;13(2):425.

5. Lee EK, Konstantinopoulos PA. Combined PARP and immune checkpoint inhibition in ovarian cancer. Trends Cancer. 2019;5(9):524-8.

6. Mouw KW, Goldberg MS, Konstantinopoulos PA, D'Andrea AD. DNA damage and repair biomarkers of immunotherapy response. Cancer Discov. 2017;7(7):675-93. 
7. Mouw KW, D'Andrea AD. DNA repair deficiency and immunotherapy response. J Clin Oncol. 2018;36(17):1710-3.

8. Pilzecker B, Buoninfante OA, Jacobs H. DNA damage tolerance in stem cells, ageing, mutagenesis, disease and cancer therapy. Nucleic Acids Res. 2019;47(14):7163-81.

9. Pilie PG, Tang C, Mills GB, Yap TA. State-of-the-art strategies for targeting the DNA damage response in cancer. Nat Rev Clin Oncol. 2019;16(2):81-104.

10. Lee EK, Konstantinopoulos PA. PARP inhibition and immune modulation: scientific rationale and perspectives for the treatment of gynecologic cancers. Ther Adv Med Oncol. 2020;12:1758835920944116.

11. Stewart RA, Pilie PG, Yap TA. Development of PARP and immune-checkpoint inhibitor combinations. Cancer Res. 2018;78(24):6717-25.

12. Turajlic S, Litchfield K, Xu H, Rosenthal R, McGranahan N, Reading J et al. Insertion-and-deletion-derived tumour-specific neoantigens and the immunogenic phenotype: a pan-cancer analysis. Lancet Oncol. 2017;18(8):1009-21.

13. Castroviejo-Bermejo M, Cruz C, Llop-Guevara A, Gutierrez-Enriquez S, Ducy M, Ibrahim YH, et al. A RAD51 assay feasible in routine tumor samples calls PARP inhibitor response beyond BRCA mutation. EMBO Mol Med. 2018;10(12):66.

14. Zhao EY, Shen Y, Pleasance E, Kasaian K, Leelakumari S, Jones M, et al. Homologous recombination deficiency and platinum-based therapy outcomes in advanced breast cancer. Clin Cancer Res. 2017;23(24):7521-30.

15. Konstantinopoulos PA, Ceccaldi R, Shapiro GI, D'Andrea AD. Homologous recombination deficiency: exploiting the fundamental vulnerability of ovarian cancer. Cancer Discov. 2015;5(11):1137-54.

16. Chopra N, Tovey H, Pearson A, Cutts R, Toms C, Proszek P, et al. Homologous recombination DNA repair deficiency and PARP inhibition activity in primary triple negative breast cancer. Nat Commun. 2020;11(1):2662.

17. Santana Dos Santos E, Lallemand F, Petitalot A, Caputo SM, Rouleau E. HRness in Breast and Ovarian Cancers. Int J Mol Sci. 2020;21(11):66.

18. Cetin B, Wabl CA, Gumusay O. The DNA damaging revolution. Crit Rev Oncol Hematol. 2020;156:103117.

19. Bryant C, Rawlinson R, Massey AJ. Chk1 inhibition as a novel therapeutic strategy for treating triple-negative breast and ovarian cancers. BMC Cancer. 2014;14:570.

20. Cleary JM, Aguirre AJ, Shapiro GI, D'Andrea AD. Biomarker-guided development of DNA repair inhibitors. Mol Cell. 2020;78(6):1070-85.

21. Torre LA, Trabert B, DeSantis CE, Miller KD, Samimi G, Runowicz CD, et al. Ovarian cancer statistics, 2018. CA Cancer J Clin. 2018:68(4):284-96.

22. Swisher EM, Lin KK, Oza AM, Scott CL, Giordano H, Sun J, et al. Rucaparib in relapsed, platinum-sensitive high-grade ovarian carcinoma (ARIEL2 Part 1): an international, multicentre, open-label, phase 2 trial. Lancet Oncol. 2017:18(1):75-87.

23. Kristeleit RS, Shapira-Frommer R, Oaknin A, Balmaña J, Ray-Coquard I, Domchek S, et al. Clinical activity of the poly (ADP-ribose) polymerase (PARP) inhibitor rucaparib in patients (pts) with high-grade ovarian carcinoma (HGOC) and a BRCA mutation (BRCAmut): Analysis of pooled data from Study 10 (parts 1, 2a, and 3) and ARIEL2 (parts 1 and 2). 2016;27(suppl_6).

24. Moore K, Colombo N, Scambia G, Kim BG, Oaknin A, Friedlander M, et al. Maintenance olaparib in patients with newly diagnosed advanced ovarian cancer. N Engl J Med. 2018;379(26):2495-505.

25. Ray-Coquard I, Pautier P, Pignata S, Perol D, Gonzalez-Martin A, Berger $\mathrm{R}$, et al. Olaparib plus bevacizumab as first-line maintenance in ovarian cancer. N Engl J Med. 2019;381(25):2416-28.

26. Ledermann J, Harter P, Gourley C, Friedlander M, Vergote I, Rustin G, et al. Olaparib maintenance therapy in platinum-sensitive relapsed ovarian cancer. N Engl J Med. 2012;366(15):1382-92.

27. Ledermann J, Harter P, Gourley C, Friedlander M, Vergote I, Rustin G, et al. Olaparib maintenance therapy in patients with platinum-sensitive relapsed serous ovarian cancer: a preplanned retrospective analysis of outcomes by BRCA status in a randomised phase 2 trial. Lancet Oncol. 2014;15(8):852-61.

28. Coleman RL, Oza AM, Lorusso D, Aghajanian C, Oaknin A, Dean A, et al. Rucaparib maintenance treatment for recurrent ovarian carcinoma after response to platinum therapy (ARIEL3): a randomised, double-blind, placebo-controlled, phase 3 trial. Lancet. 2017;390(10106):1949-61.
29. Mirza MR, Monk B t, Herrstedt J Oza AM Mahner S, Redondo A et al. Niraparib maintenance therapy in platinum-sensitive, recurrent ovarian cancer. N Engl J Med. 2016;375(22):2154-64.

30. Barkal AA, Brewer RE, Markovic M, Kowarsky M, Barkal SA, Zaro BW, et al. CD24 signalling through macrophage Siglec-10 is a target for cancer immunotherapy. Nature. 2019;572(7769):392-6.

31. Timperi E, Vissio E, Marchio C, Romano E. The immune landscape in women cancers. Cancer Treat Res. 2020;180:215-49.

32. Robson M, Im SA, Senkus E, Xu B, Domchek SM, Masuda N, et al. Olaparib for metastatic breast cancer in patients with a germline BRCA mutation. N Engl J Med. 2017;377(6):523-33.

33. Litton JK, Rugo HS, Ettl J, Hurvitz SA, Goncalves A, Lee KH, et al. Talazoparib in patients with advanced breast cancer and a germline BRCA mutation. N Engl J Med. 2018;379(8):753-63.

34. Takahashi N, Surolia I, Thomas A. Targeting DNA repair to drive immune responses: it's time to reconsider the strategy for clinical translation. Clin Cancer Res. 2020;6:66

35. Yap TA, Plummer R, Azad NS, Helleday T. The DNA damaging revolution: PARP inhibitors and beyond. Am Soc Clin Oncol Educ Book. 2019:39:185-95.

36. Min A, Im SA. PARP inhibitors as therapeutics: beyond modulation of PARylation. Cancers. 2020;12(2):66.

37. He Q, Jiang X, Zhou X, Weng J. Targeting cancers through TCR-peptide/ MHC interactions. J Hematol Oncol. 2019:12(1):139.

38. Yang Q, Cao W, Wang Z, Zhang B, Liu J. Regulation of cancer immune escape: the roles of miRNAs in immune checkpoint proteins. Cancer Lett. 2018:431:73-84.

39. Esensten JH, Helou YA, Chopra G, Weiss A, Bluestone JA. CD28 costimulation: from mechanism to therapy. Immunity. 2016;44(5):973-88.

40. Qin S, Xu L, Yi M, Yu S, Wu K, Luo S. Novel immune checkpoint targets: moving beyond PD-1 and CTLA-4. Mol Cancer. 2019;18(1):155.

41. Topalian SL, Drake CG, Pardoll DM. Immune checkpoint blockade: a common denominator approach to cancer therapy. Cancer Cell. 2015;27(4):450-61.

42. Greenwald RJ, Freeman GJ, Sharpe AH. The B7 family revisited. Annu Rev Immunol. 2005;23:515-48.

43. Nallasamy P, Chava S, Verma SS, Mishra S, Gorantla S, Coulter DW, et al. PD-L1, inflammation, non-coding RNAs, and neuroblastoma: Immunooncology perspective. Semin Cancer Biol. 2018;52(Pt 2):53-65.

44. Fukumura D, Kloepper J, Amoozgar Z, Duda DG, Jain RK. Enhancing cancer immunotherapy using antiangiogenics: opportunities and challenges. Nat Rev Clin Oncol. 2018;15(5):325-40.

45. Keenan TE, Tolaney SM. Role of immunotherapy in triple-negative breast cancer. J Natl Compr Canc Netw. 2020;18(4):479-89.

46. Yang C, Xia BR, Zhang ZC, Zhang YJ, Lou G, Jin WL. Immunotherapy for ovarian cancer: adjuvant, combination, and neoadjuvant. Front Immunol. 2020;11:577-869.

47. van Dijk N, Funt SA, Blank CU, Powles T, Rosenberg JE, van der Heijden MS. The cancer immunogram as a framework for personalized immunotherapy in urothelial cancer. Eur Urol. 2019;75(3):435-44.

48. Park S, Lee H, Lee B, Lee SH, Sun JM, Park WY, et al. DNA damage response and repair pathway alteration and its association with tumor mutation burden and platinum-based chemotherapy in SCLC. JThorac Oncol. 2019;14(9):1640-50.

49. Li A, Yi M, Qin S, Chu Q, Luo S, Wu K. Prospects for combining immune checkpoint blockade with PARP inhibition. J Hematol Oncol. 2019:12(1):98

50. Shevtsov M, Sato H, Multhoff G, Shibata A. novel approaches to improve the efficacy of immuno-radiotherapy. Front Oncol. 2019:9:156.

51. Dillon MT, Bergerhoff KF, Pedersen M, Whittock H, Crespo-Rodriguez $\mathrm{E}$, Patin EC, et al. ATR inhibition potentiates the radiationinduced inflammatory tumor microenvironment. Clin Cancer Res. 2019;25(11):3392-403.

52. Sen T, Della Corte CM, Milutinovic S, Cardnell RJ, Diao L, Ramkumar K et al. Combination treatment of the oral CHK1 inhibitor, SRA737, and low-dose gemcitabine enhances the effect of programmed death ligand 1 blockade by modulating the immune microenvironment in SCLC. J Thorac Oncol. 2019:14(12):2152-63.

53. Skoulidis F, Goldberg ME, Greenawalt DM, Hellmann MD, Awad MM, Gainor JF, et al. STK11/LKB1 mutations and PD-1 inhibitor resistance in KRAS-mutant lung adenocarcinoma. Cancer Discov. 2018;8(7):822-35. 
54. Ricciuti B, Recondo G, Spurr LF, Li YY, Lamberti G, Venkatraman D, et al. Impact of DNA damage response and repair (DDR) gene mutations on efficacy of PD-(L)1 immune checkpoint inhibition in non-small cell lung cancer. Clin Cancer Res. 2020;6:66.

55. Havel JJ, Chowell D, Chan TA. The evolving landscape of biomarkers for checkpoint inhibitor immunotherapy. Nat Rev Cancer. 2019;19(3):133-50.

56. Mandal R, Samstein RM, Lee KW, Havel JJ, Wang H, Krishna C, et al. Genetic diversity of tumors with mismatch repair deficiency influences anti-PD-1 immunotherapy response. Science. 2019;364(6439):485-91.

57. Chalabi M, Fanchi LF, Dijkstra KK, Van den Berg JG, Aalbers AG, Sikorska K, et al. Neoadjuvant immunotherapy leads to pathological responses in MMR-proficient and MMR-deficient early-stage colon cancers. Nat Med. 2020;26(4):566-76.

58. Alexandrov LB, Nik-Zainal S, Wedge DC, Aparicio SA, Behjati S, Biankin $\mathrm{AV}$, et al. Signatures of mutational processes in human cancer. Nature. 2013;500(7463):415-21.

59. McGranahan N, Furness AJ, Rosenthal R, Ramskov S, Lyngaa R, Saini SK, et al. Clonal neoantigens elicit T cell immunoreactivity and sensitivity to immune checkpoint blockade. Science. 2016;351(6280):1463-9.

60. Miao D, Margolis CA, Vokes NI, Liu D, Taylor-Weiner A, Wankowicz SM, et al. Genomic correlates of response to immune checkpoint blockade in microsatellite-stable solid tumors. Nat Genet. 2018;50(9):1271-81.

61. Soo RA. Shedding light on the molecular determinants of response to anti-PD-1 therapy. Transl Lung Cancer Res. 2015;4(6):816-9.

62. Conway JR, Kofman E, Mo SS, Elmarakeby H, Van Allen E. Genomics of response to immune checkpoint therapies for cancer: implications for precision medicine. Genome Med. 2018;10(1):93.

63. Tang F, Zheng P. Tumor cells versus host immune cells: whose PD-L1 contributes to PD-1/PD-L1 blockade mediated cancer immunotherapy? Cell Biosci. 2018:8:34

64. Lai Q, Wang H, Li A, Xu Y, Tang L, Chen Q, et al. Decitibine improve the efficiency of anti-PD-1 therapy via activating the response to IFN/PD-L1 signal of lung cancer cells. Oncogene. 2018;37(17):2302-12.

65. Salgado R, Denkert C, Demaria S, Sirtaine N, Klauschen F, Pruneri G, et al. The evaluation of tumor-infiltrating lymphocytes (TILs) in breast cancer: recommendations by an International TILs Working Group 2014. Ann Oncol. 2015;26(2):259-71.

66. Woroniecka Kl, Rhodin KE, Chongsathidkiet P, Keith KA, Fecci PE. T-cell dysfunction in glioblastoma: applying a new framework. Clin Cancer Res. 2018;24(16):3792-802.

67. Zhu J, Petit PF, Van den Eynde BJ. Apoptosis of tumor-infiltrating $T$ lymphocytes: a new immune checkpoint mechanism. Cancer Immunol Immunother. 2019;68(5):835-47.

68. Cao D, Zhao J, Nguyan LN, Nguyen LNT, Khanal S, Dang X, et al. Disruption of telomere integrity and DNA repair machineries by KML001 induces T cell senescence, apoptosis, and cellular dysfunctions. Front Immunol. 2019;10:1152.

69. Boulares $A H$, Yakovlev AG, Ivanova V, Stoica BA, Wang G, lyer S, et al. Role of poly(ADP-ribose) polymerase (PARP) cleavage in apoptosis. Caspase 3-resistant PARP mutant increases rates of apoptosis in transfected cells. J Biol Chem. 1999;274(33):22932-40.

70. Brown JS, Sundar R, Lopez J. Combining DNA damaging therapeutics with immunotherapy: more haste, less speed. Br J Cancer. 2018;118(3):312-24.

71. Aptsiauri N, Ruiz-Cabello F, Garrido F. The transition from HLA-I positive to HLA-I negative primary tumors: the road to escape from T-cell responses. Curr Opin Immunol. 2018;51:123-32.

72. Mu C, Zhang X, Wang L, Xu A, Ahmed KA, Pang X, et al. Enhanced suppression of polyclonal CD8(+)25(+) regulatory T cells via exosomal arming of antigen-specific peptide/MHC complexes. J Leukoc Biol. 2017;101(5):1221-31.

73. Vendetti FP, Karukonda P, Clump DA, Teo T, Lalonde R, Nugent K, et al. ATR kinase inhibitor AZD6738 potentiates CD8+ T cell-dependent antitumor activity following radiation. J Clin Invest. 2018;128(9):3926-40.

74. Wang Y, Lin YX, Qiao SL, An HW, Ma Y, Qiao ZY, et al. Polymeric nanoparticles promote macrophage reversal from M2 to M1 phenotypes in the tumor microenvironment. Biomaterials. 2017;112:153-63.

75. Mantovani A, Marchesi F, Malesci A, Laghi L, Allavena P. Tumourassociated macrophages as treatment targets in oncology. Nat Rev Clin Oncol. 2017;14(7):399-416.
76. Stiff A, Trikha P, Wesolowski R, Kendra K, Hsu V, Uppati S, et al. Myeloidderived suppressor cells express Bruton's Tyrosine kinase and can be depleted in tumor-bearing hosts by ibrutinib treatment. Cancer Res. 2016;76(8):2125-36.

77. Kobayashi Y, Lim SO, Yamaguchi H. Oncogenic signaling pathways associated with immune evasion and resistance to immune checkpoint inhibitors in cancer. Semin Cancer Biol. 2019;6:66.

78. Liu P, Cheng H, Roberts TM, Zhao JJ. Targeting the phosphoinositide 3-kinase pathway in cancer. Nat Rev Drug Discov. 2009;8(8):627-44.

79. Millis SZ, Ikeda S, Reddy S, Gatalica Z, Kurzrock R. Landscape of phosphatidylinositol-3-kinase pathway alterations across 19784 diverse solid tumors. JAMA Oncol. 2016;2(12):1565-73.

80. Bian X, Gao J, Luo F, Rui C, Zheng T, Wang D, et al. PTEN deficiency sensitizes endometrioid endometrial cancer to compound PARPPI3K inhibition but not PARP inhibition as monotherapy. Oncogene. 2018;37(3):341-51.

81. McCabe N, Hanna C, Walker SM, Gonda D, Li J, Wikstrom K, et al. Mechanistic rationale to target PTEN-deficient tumor cells with inhibitors of the DNA damage response kinase ATM. Cancer Res. 2015;75(11):2159-65.

82. Kitajima S, Ivanova E, Guo S, Yoshida R, Campisi M, Sundararaman SK, et al. Suppression of STING associated with LKB1 Loss in KRAS-driven lung cancer. Cancer Discov. 2019;9(1):34-45.

83. Tolba MF, Omar HA. Immunotherapy, an evolving approach for the management of triple negative breast cancer: converting nonresponders to responders. Crit Rev Oncol Hematol. 2018;122:202-7.

84. McLaughlin M, Patin EC, Pedersen M, Wilkins A, Dillon MT, Melcher AA et al. Inflammatory microenvironment remodelling by tumour cells after radiotherapy. Nat Rev Cancer. 2020;20(4):203-17.

85. Keenan TE, Burke KP, Van Allen EM. Genomic correlates of response to immune checkpoint blockade. Nat Med. 2019;25(3):389-402.

86. Ribas A, Wolchok JD. Cancer immunotherapy using checkpoint blockade. Science. 2018;359(6382):1350-5.

87. Strickland KC, Howitt BE, Shukla SA, Rodig S, Ritterhouse LL, Liu JF, et al. Association and prognostic significance of BRCA1/2-mutation status with neoantigen load, number of tumor-infiltrating lymphocytes and expression of PD-1/PD-L1 in high grade serous ovarian cancer. Oncotarget. 2016;7(12):13587-98.

88. Min A, Kim K, Jeong K, Choi S, Kim S, Suh KJ, et al. Homologous repair deficiency score for identifying breast cancers with defective DNA damage response. Sci Rep. 2020;10(1):12506.

89. Denkert C, Liedtke C, Tutt A, von Minckwitz G. Molecular alterations in triple-negative breast cancer-the road to new treatment strategies. Lancet. 2017;389(10087):2430-42.

90. Milanesio MC, Giordano S, Valabrega G. Clinical implications of DNA repair defects in high-grade serous ovarian carcinomas. Cancers. 2020;12(5):66.

91. Le DT, Durham JN, Smith KN, Wang H, Bartlett BR, Aulakh LK, et al. Mismatch repair deficiency predicts response of solid tumors to PD-1 blockade. Science. 2017;357(6349):409-13.

92. Le DT, Uram JN, Wang $H$, Bartlett BR, Kemberling $H$, Eyring AD, et al. PD-1 Blockade in tumors with mismatch-repair deficiency. N Engl J Med. 2015:372(26):2509-20.

93. Davies H, Morganella S, Purdie CA, Jang SJ, Borgen E, Russnes H, et al. Whole-genome sequencing reveals breast cancers with mismatch repair deficiency. Cancer Res. 2017;77(18):4755-62.

94. Howitt BE, Strickland KC, Sholl LM, Rodig S, Ritterhouse LL, Chowdhury D, et al. Clear cell ovarian cancers with microsatellite instability: a unique subset of ovarian cancers with increased tumor-infiltrating lymphocytes and PD-1/PD-L1 expression. Oncoimmunology. 2017;6(2):e1277308.

95. Ioannidou A, Goulielmaki E, Garinis GA. DNA damage: from chronic inflammation to age-related deterioration. Front Genet. 2016;7:187.

96. McGrail DJ, Federico L, Li Y, Dai H, Lu Y, Mills GB, et al. Multi-omics analysis reveals neoantigen-independent immune cell infiltration in copy-number driven cancers. Nat Commun. 2018;9(1):1317.

97. Jiao S, Xia W, Yamaguchi H, Wei Y, Chen MK, Hsu JM, et al. PARP inhibitor upregulates PD-L1 expression and enhances cancer-associated immunosuppression. Clin Cancer Res. 2017;23(14):3711-20. 
98. Maya-Mendoza A, Moudry P, Merchut-Maya JM, Lee M, Strauss R, Bartek J. High speed of fork progression induces DNA replication stress and genomic instability. Nature. 2018;559(7713):279-84.

99. Sato $H$, Jeggo PA, Shibata A. Regulation of programmed death-ligand 1 expression in response to DNA damage in cancer cells: implications for precision medicine. Cancer Sci. 2019;110(11):3415-23.

100. Pantelidou C, Sonzogni O, De Oliveria TM, Mehta AK, Kothari A, Wang $D$, et al. PARP inhibitor efficacy depends on CD8(+) T-cell recruitment via intratumoral STING pathway activation in BRCA-deficient models of triple-negative breast cancer. Cancer Discov. 2019;9(6):722-37.

101. Parkes EE, Walker SM, Taggart LE, McCabe N, Knight LA, Wilkinson R, et al. Activation of STING-dependent innate immune signaling by S-phase-specific DNA damage in breast cancer. J Natl Cancer Inst. 2017;109(1):66.

102. Ding L, Kim HJ, Wang Q, Kearns M, Jiang T, Ohlson CE, et al. PARP inhibition elicits STING-dependent antitumor immunity in Brca 1deficient ovarian cancer. Cell Rep. 2018;25(11):2972-80e5.

103. Zhang C, Shang G, Gui X, Zhang X, Bai XC, Chen ZJ. Structural basis of STING binding with and phosphorylation by TBK1. Nature. 2019:567(7748):394-8.

104. Wu J, Sun L, Chen X, Du F, Shi H, Chen C, et al. Cyclic GMP-AMP is an endogenous second messenger in innate immune signaling by cytosolic DNA. Science. 2013;339(6121):826-30.

105. Sato H, Niimi A, Yasuhara T, Permata TBM, Hagiwara Y, Isono M, et al. DNA double-strand break repair pathway regulates PD-L1 expression in cancer cells. Nat Commun. 2017;8(1):1751.

106. Robillard L, Nguyen M, Loehr A, Orsulic S, Kristeleit RS, Lin K, et al. Preclinical evaluation of the PARP inhibitor rucaparib in combination with PD-1 and PD-L1 inhibition in a syngeneic BRCA1 mutant ovarian cancer model. AACR. 2017;6:66.

107. Wang Z, Sun K, Xiao Y, Feng B, Mikule K, Ma X, et al. Niraparib activates interferon signaling and potentiates anti-PD-1 antibody efficacy in tumor models. Sci Rep. 2019;9(1):1853.

108. Sun LL, Yang RY, Li CW, Chen MK, Shao B, Hsu JM, et al. Inhibition of ATR downregulates PD-L1 and sensitizes tumor cells to T cell-mediated killing. Am J Cancer Res. 2018;8(7):1307-16.

109. Higuchi T, Flies DB, Marjon NA, Mantia-Smaldone G, Ronner L, Gimotty PA, et al. CTLA-4 Blockade synergizes therapeutically with PARP inhibition in BRCA1-deficient ovarian cancer. Cancer Immunol Res. 2015;3(11):1257-68.

110. Das R, Verma R, Sznol M, Boddupalli CS, Gettinger SN, Kluger H, et al. Combination therapy with anti-CTLA-4 and anti-PD-1 leads to distinct immunologic changes in vivo. J Immunol. 2015;194(3):950-9.

111. Shen Y, Aoyagi-Scharber M, Wang B. Trapping poly(ADP-Ribose) polymerase. J Pharmacol Exp Ther. 2015;353(3):446-57.

112. Leo E, Johannes J, Illuzzi G, Zhang A, Hemsley P, Bista MJ, et al. Abstract LB-273: A head-to-head comparison of the properties of five clinical PARP inhibitors identifies new insights that can explain both the observed clinical efficacy and safety profiles. AACR. 2018;6:66.

113. Wengner AM, Kirchhoff D, Roese L, Berndt S, Siemeister G, Kreft B, et al. Synergistic activity of the ATR inhibitor BAY 1895344 in combination with immune checkpoint inhibitors in preclinical tumor models. AACR. 2019;6:66.

114. Fehlings M, Simoni Y, Penny HL, Becht E, Loh CY, Gubin MM, et al. Checkpoint blockade immunotherapy reshapes the high-dimensional phenotypic heterogeneity of murine intratumoural neoantigen-specific CD8(+) T cells. Nat Commun. 2017;8(1):562.

115. Harding SM, Benci JL, Irianto J, Discher DE, Minn AJ, Greenberg RA. Mitotic progression following DNA damage enables pattern recognition within micronuclei. Nature. 2017;548(7668):466-70.

116. Qiu Z, Oleinick NL, Zhang J. ATR/CHK1 inhibitors and cancer therapy. Radiother Oncol. 2018;126(3):450-64.

117. Gray HJ, Bell-McGuinn K, Fleming GF, Cristea M, Xiong H, Sullivan D, et al. Phase I combination study of the PARP inhibitor veliparib plus carboplatin and gemcitabine in patients with advanced ovarian cancer and other solid malignancies. Gynecol Oncol. 2018;148(3):507-14.

118. Tian H, Gao Z, Li H, Zhang B, Wang G, Zhang Q, et al. DNA damage response-a double-edged sword in cancer prevention and cancer therapy. Cancer Lett. 2015;358(1):8-16.
119. Michelena J, Gatti M, Teloni F, Imhof R, Altmeyer M. Basal CHK1 activity safeguards its stability to maintain intrinsic S-phase checkpoint functions. J Cell Biol. 2019;218(9):2865-75.

120. Bradbury A, Hall S, Curtin N, Drew Y. Targeting ATR as Cancer Therapy: A new era for synthetic lethality and synergistic combinations? Pharmacol Ther. 2020;207:107450.

121. Sen T, Gay CM, Byers LA. Targeting DNA damage repair in small cell lung cancer and the biomarker landscape. Transl Lung Cancer Res. 2018;7(1):50-68.

122. Bever KM, Le DT. DNA repair defects and implications for immunotherapy. J Clin Invest. 2018;128(10):4236-42.

123. Rodriguez Ml, Majuelos-Melguizo J, Marti Martin-Consuegra JM, Ruiz de Almodovar M, Lopez-Rivas A, Javier Oliver F. Deciphering the insights of poly(ADP-ribosylation) in tumor progression. Med Res Rev. 2015;35(4):678-97.

124. Wang WY, Cao YX, Zhou X, Wei B, Zhan L, Fu LT. HMGA2 gene silencing reduces epithelial-mesenchymal transition and lymph node metastasis in cervical cancer through inhibiting the ATR/Chk1 signaling pathway. Am J Transl Res. 2018;10(10):3036-52.

125. Galati S, Boni C, Gerra MC, Lazzaretti M, Buschini A. Autophagy: a player in response to oxidative stress and DNA damage. Oxid Med Cell Longev. 2019;2019:5692958.

126. Vanzo R, Bartkova J, Merchut-Maya JM, Hall A, Bouchal J, Dyrskjot L, et al. Autophagy role(s) in response to oncogenes and DNA replication stress. Cell Death Differ. 2020;27(3):1134-53.

127. Ji Y, Wang Q, Zhao Q, Zhao S, Li L, Sun G, et al. Autophagy suppression enhances DNA damage and cell death upon treatment with PARP inhibitor Niraparib in laryngeal squamous cell carcinoma. Appl Microbiol Biotechnol. 2019;103(23-24):9557-68.

128. Bellei B, Picardo M. Premature cell senescence in human skin: Dual face in chronic acquired pigmentary disorders. Ageing Res Rev. 2020;57:100981.

129. Fleury H, Malaquin N, Tu V, Gilbert S, Martinez A, Olivier MA, et al. Exploiting interconnected synthetic lethal interactions between PARP inhibition and cancer cell reversible senescence. Nat Commun. 2019;10(1):2556.

130. Bertran-Alamillo J, Cattan V, Schoumacher M, Codony-Servat J, Gimenez-Capitan A, Cantero F, et al. AURKB as a target in non-small cell lung cancer with acquired resistance to anti-EGFR therapy. Nat Commun. 2019;10(1):1812.

131. Green AR, Aleskandarany MA, Ali R, Hodgson EG, Atabani S, De Souza K, et al. Clinical impact of tumor DNA repair expression and T-cell Infiltration in breast cancers. Cancer Immunol Res. 2017;5(4):292-9.

132. Marti JM, Fernandez-Cortes M, Serrano-Saenz S, Zamudio-Martinez E, Delgado-Bellido D, Garcia-Diaz A, et al. The multifactorial role of PARP-1 in tumor microenvironment. Cancers. 2020;12(3):66.

133. Karzai F, Madan RA, Owens H, Couvillon A, Hankin A, Williams M, et al. A phase 2 study of olaparib and durvalumab in metastatic castrateresistant prostate cancer (mCRPC) in an unselected population. Am Soc Clin Oncol. 2018;6:66.

134. Lee JM, Cimino-Mathews A, Peer CJ, Zimmer A, Lipkowitz S, Annunziata $C M$, et al. Safety and clinical activity of the programmed death-ligand 1 inhibitor durvalumab in combination with poly(ADP-Ribose) polymerase inhibitor olaparib or vascular endothelial growth factor receptor 1-3 inhibitor cediranib in women's cancers: a dose-escalation. Phase I Study J Clin Oncol. 2017;35(19):2193-202.

135. Thomas A, Vilimas R, Trindade C, Erwin-Cohen R, Roper N, Xi L, et al. Durvalumab in combination with olaparib in patients with relapsed SCLC: results from a phase II study. J Thorac Oncol. 2019;14(8):1447-57.

136. Konstantinopoulos PA, Waggoner SE, Vidal GA, Mita MM, Fleming GF, Holloway RW, et al. TOPACIO/Keynote-162 (NCT02657889): a phase 1/2 study of niraparib + pembrolizumab in patients (pts) with advanced triple-negative breast cancer or recurrent ovarian cancer (ROC) - -results from ROC cohort. Am Soc Clin Oncol. 2018;6:66.

137. Yu EY, Massard C, Retz M, Tafreshi A, Carles Galceran J, Hammerer P, et al. Keynote-365 cohort a: pembrolizumab (pembro) plus olaparib in docetaxel-pretreated patients (pts) with metastatic castrate-resistant prostate cancer (mCRPC). Am Soc Clin Oncol. 2019;6:66.

138. Friedlander M, Meniawy T, Markman B, Mileshkin LR, Harnett PR, Millward $\mathrm{M}$, et al. A phase $1 \mathrm{~b}$ study of the anti-PD-1 monoclonal antibody 
BGB-A317 (A317) in combination with the PARP inhibitor BGB-290 (290) in advanced solid tumors. Am Soc Clin Oncol. 2017;6:66.

139. Yap T, Krebs MG, Postel-Vinay S, Bang Y, El-Khoueiry A, Abida W, et al. Phase I modular study of AZD6738, a novel oral, potent and selective ataxia telangiectasia Rad3-related (ATR) inhibitor in combination (combo) with carboplatin, olaparib or durvalumab in patients (pts) with advanced cancers. 2016;69:S2.

140. Konstantinopoulos P, Munster P, Forero-Torez A, Holloway R, Schwartzberg L, Matulonis U, et al. Topacio: preliminary activity and safety in patients (pts) with platinum-resistant ovarian cancer (PROC) in a phase $1 / 2$ study of niraparib in combination with pembrolizumab. AACR. 2018;149:246.

141. Domchek SM, Postel-Vinay S, Bang Y-J, Park YH, Alexandre J, Delord J-P, et al. Abstract PD6-11: an open-label, multitumor, phase II basket study of olaparib and durvalumab (MEDIOLA): Results in germline BRCAmutated (gBRCAm) HER2-negative metastatic breast cancer (MBC). AACR. 2018;6:66.

142. Drew Y, De Jonge M, Hong S, Park Y, Wolfer A, Brown J, et al. An openlabel, phase II basket study of olaparib and durvalumab (MEDIOLA): results in germline BRCA-mutated (gBRCAm) platinum-sensitive relapsed (PSR) ovarian cancer (OC). AACR. 2018;149:246-7.

143. Cesaire M, Thariat J, Candeias SM, Stefan D, Saintigny Y, Chevalier F. Combining PARP inhibition, radiation, and immunotherapy: a possible strategy to improve the treatment of cancer? Int J Mol Sci. 2018;19(12):66.

144. Patel P, Sun L, Robbins Y, Clavijo PE, Friedman J, Silvin C, et al. Enhancing direct cytotoxicity and response to immune checkpoint blockade following ionizing radiation with Wee1 kinase inhibition. Oncoimmunology. 2019;8(11):e1638207.

145. Prasanna T, Wu F, Khanna KK, Yip D, Malik L, Dahlstrom JE, et al. Optimizing poly (ADP-ribose) polymerase inhibition through combined epigenetic and immunotherapy. Cancer Sci. 2018;109(11):3383-92.

146. Lajos Pusztai HSH, Christina Yau, Denise Wolf, Anne M. Wallace, Rebecca Shatsky, Teresa Helsten, Judy C. Boughey, Tufia Haddad, Erica StringerReasor, Carla Falkson, A. Jo Chien, Rita Mukhtar, Anthony Elias, Borges Virginia, Rita Nanda, Douglas Yee, Kevin Kalinsky, Kathy S. Albain, Aixa Soyano Muller, Kathleen Kemmer, Amy S. Clark, Claudine Isaacs, Alexandra Thomas, Nola Hylton, W. Fraser Symmans, Jane Perlmutter, Michelle Melisko, Hope S. Rugo, Richard Schwab, Amy Wilson, Amy Wilson, Ruby Singhrao, Smita Asare, Laura J. Van't Veer, Angela M. DeMichele, Ashish Sanil, Donald A. Berry, Laura J. Evaluation of durvalumab in combination with olaparib and paclitaxel in high-risk HER2 negative stage II/III breast cancer: Results from the I-SPY 2 TRIAL. AACR; 2020.

147. Farkkila A, Gulhan DC, Casado J, Jacobson CA, Nguyen H, Kochupurakkal $B$, et al. Immunogenomic profiling determines responses to combined PARP and PD-1 inhibition in ovarian cancer. Nat Commun. 2020;11(1):1459.

148. Sharma P. Biology and management of patients with triple-negative breast cancer. Oncologist. 2016;21(9):1050-62.

149. Sokol ES, Pavlick D, Khiabanian H, Frampton GM, Ross JS, Gregg JP, et al. Pan-cancer analysis of BRCA1 and BRCA2 genomic alterations and their association with genomic instability as measured by genome-wide loss of heterozygosity. JCO Precis Oncol. 2020:4:442-65.

150. Shen J, Zhao W, Ju Z, Wang L, Peng Y, Labrie M, et al. PARPi triggers the STING-dependent immune response and enhances the therapeutic efficacy of immune checkpoint blockade independent of BRCAness. Cancer Res. 2019;79(2):311-9.

151. Sen T, Rodriguez BL, Chen L, Corte CMD, Morikawa N, Fujimoto J, et al. Targeting DNA damage response promotes antitumor immunity through STING-mediated T-cell activation in small cell lung cancer. Cancer Discov. 2019;9(5):646-61.

\section{Publisher's Note}

Springer Nature remains neutral with regard to jurisdictional claims in published maps and institutional affiliations.

Ready to submit your research? Choose BMC and benefit from:

- fast, convenient online submission

- thorough peer review by experienced researchers in your field

- rapid publication on acceptance

- support for research data, including large and complex data types

- gold Open Access which fosters wider collaboration and increased citations

- maximum visibility for your research: over 100M website views per year

At BMC, research is always in progress.

Learn more biomedcentral.com/submissions 